\title{
Orbit-orbit relativistic correction calculated with all-electron molecular explicitly correlated Gaussians
}

Monika Stanke, Ewa Palikot, Dariusz Kẹdziera, and Ludwik Adamowicz

Citation: The Journal of Chemical Physics 145, 224111 (2016); doi: 10.1063/1.4971376

View online: http://dx.doi.org/10.1063/1.4971376

View Table of Contents: http://aip.scitation.org/toc/jcp/145/22

Published by the American Institute of Physics

\section{Articles you may be interested in}

Convergence of coupled cluster perturbation theory

The Journal of Chemical Physics 145, 224104224104 (2016); 10.1063/1.4971294

Outstanding performance of configuration interaction singles and doubles using exact exchange Kohn-Sham orbitals in real-space numerical grid method

The Journal of Chemical Physics 145, 224309224309 (2016); 10.1063/1.4971786

Perspective: Methods for large-scale density functional calculations on metallic systems

The Journal of Chemical Physics 145, 220901220901 (2016); 10.1063/1.4972007

Analytic second derivatives from auxiliary density perturbation theory

The Journal of Chemical Physics 145, 224103224103 (2016); 10.1063/1.4971292

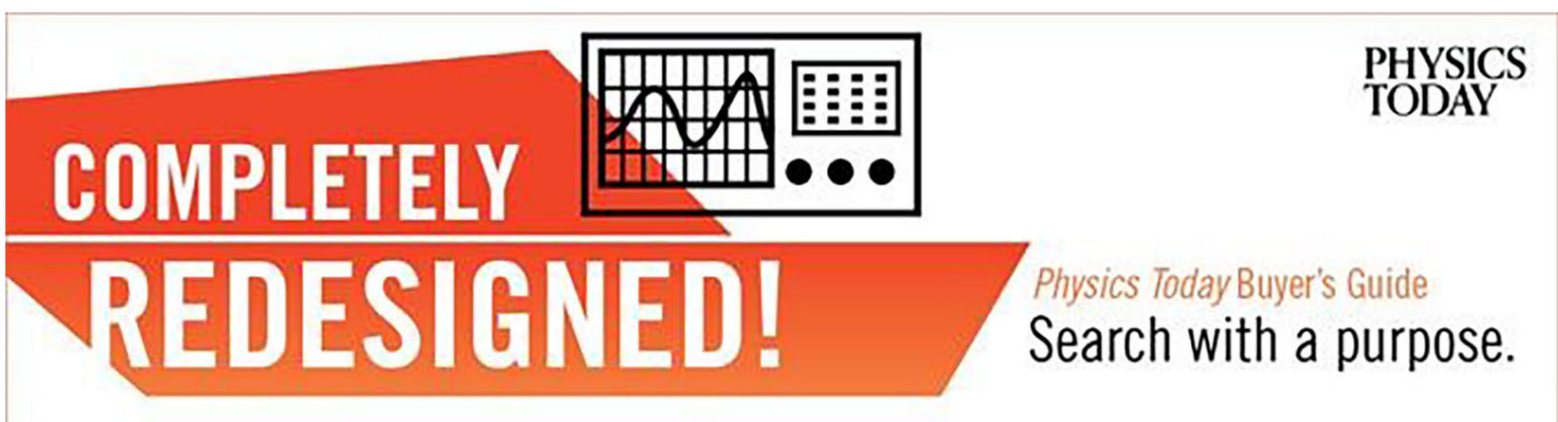




\title{
Orbit-orbit relativistic correction calculated with all-electron molecular explicitly correlated Gaussians
}

\author{
Monika Stanke, ${ }^{1, a)}$ Ewa Palikot, ${ }^{1}$ Dariusz Kȩdziera, ${ }^{2}$ and Ludwik Adamowicz ${ }^{3, b)}$ \\ ${ }^{1}$ Institute of Physics, Faculty of Physics, Astronomy, and Informatics, Nicolaus Copernicus University in Torun, \\ ul. Grudziadzka 5, Toruń PL 87-100, Poland \\ ${ }^{2}$ Faculty of Chemistry, Nicolaus Copernicus University, ul. Gagarina 7, Toruń PL 87-100, Poland \\ ${ }^{3}$ Department of Chemistry and Biochemistry and Department of Physics, University of Arizona, \\ Tucson, Arizona 85721, USA and Interdisciplinary Center for Modern Technologies, \\ Nicolaus Copernicus University, ul. Wileńska 4, Torun PL 87-100, Poland
}

(Received 30 September 2016; accepted 22 November 2016; published online 15 December 2016)

\begin{abstract}
An algorithm for calculating the first-order electronic orbit-orbit magnetic interaction correction for an electronic wave function expanded in terms of all-electron explicitly correlated molecular Gaussian (ECG) functions with shifted centers is derived and implemented. The algorithm is tested in calculations concerning the $\mathrm{H}_{2}$ molecule. It is also applied in calculations for $\mathrm{LiH}$ and $\mathrm{H}_{3}^{+}$molecular systems. The implementation completes our work on the leading relativistic correction for ECGs and paves the way for very accurate ECG calculations of ground and excited potential energy surfaces (PESs) of small molecules with two and more nuclei and two and more electrons, such as $\mathrm{HeH}^{-}, \mathrm{H}_{3}^{+}$, $\mathrm{HeH}_{2}^{+}$, and $\mathrm{LiH}_{2}^{+}$. The PESs will be used to determine rovibrational spectra of the systems. Published by AIP Publishing. [http://dx.doi.org/10.1063/1.4971376]
\end{abstract}

\section{INTRODUCTION}

The majority of our knowledge of the astronomical world is derived from the emission, absorption, and scattering of electromagnetic radiation from atoms and molecules. The astronomical observations of atomic and molecular spectra including transition energies, intensities, and light polarization complemented with laboratory experiments and theoretical calculations are essential in the study of stellar atmospheres. ${ }^{1}$ A wealth of information on the stellar media is contained in the intensities, widths, and polarizations of the spectral transitions. These properties are key inputs to computing the opacity of stars. The transition strengths depend on the transition electric and magnetic dipole moments, as well as on the transition electric quadruple moments. Also forbidden transitions which are strongly suppressed at higher density such as in laboratory plasmas are often seen in lower density situations where the forbidden lines can be as strong as or even stronger than the allowed ones. Accurate atomic and molecular calculations can provide values of the mentioned properties and allow assigning the spectral lines to specific rovibrational transitions.

The breadth and accuracy of rovibrational spectroscopy of small neutral and ionic molecular systems have been steadily improving due to development of new experimental methods for laser generation and frequency metrology. ${ }^{2}$ The data collected using those new techniques are beginning to reveal deviations with the most accurate theoretical calculations suggesting that the accuracy of the existing quantum-mechanical methods is no longer adequate. Particularly calculations concerning molecules with more than two electrons and/or two nuclei have not been as accurate as the measurements. For

\footnotetext{
a)Electronic address: monika@fizyka.umk.pl

b) Electronic address: ludwik@email.arizona.edu
}

example, our recent calculations of the vibrational spectrum of the $\mathrm{H}_{3}^{+}$ion, ${ }^{3-6}$ by far the most accurate ever performed, only reached the average accuracy of about $0.1 \mathrm{~cm}^{-1}$ in reproducing the experimental rovibrational frequencies. A part of the reason the $\mathrm{H}_{3}^{+}$calculations did not achieve a higher accuracy was an incomplete treatment of the relativistic corrections. Even though the $\mathrm{H}_{3}^{+}$potential energy surface was calculated in almost 40000 points using 900 explicitly correlated Gaussian (ECG) functions with shifted centers to expand the electronic wave function, the relativistic corrections taken from the work of Cencek and Kutzelnigg ${ }^{7}$ were only calculated in 69 points.

To correct this shortcoming, we have recently initiated an effort to derive and implement algorithms for calculating the leading relativistic and quantum electrodynamic (QED) corrections for an electronic wave function representing a molecular system with arbitrary numbers of electrons and nuclei expanded in terms of all-electron ECGs. ${ }^{8}$ At first, we consider ECGs without angular factors (i.e., no nodes at the nuclei). For such functions, we recently derived algorithms for calculating matrix elements involving the mass-velocity (MV) and Darwin (D) relativistic operators. In this work, we consider the operator representing the orbit-orbit $(\mathrm{OO})$ magnetic interaction.

The procedure for calculating the leading relativistic corrections is based on the first order of the perturbation theory and involves the standard expectation-value calculation of the relativistic operator ( $\mathrm{MV}+\mathrm{D}+\mathrm{OO}$ ) with the wave function representing the considered state of the system. In our calculations, the wave function for each PES point is obtained in variational calculations involving extensive optimization of all non-linear parameters of the ECGs. In this optimization, the analytical energy gradient determined with respect to these parameters is employed. The gradient-based 
approach enables to generate a large, very well optimized ECG basis set for each considered state (ground or excited) of the system and provides very accurate state wave function and energy.

The implementation of the electronic orbit-orbit corrections will allow recalculation of the $\mathrm{H}_{3}^{+} 40$ 000-point PES with the complete leading relativistic correction determined at each PES point. With this the upper part of the rovibrational spectrum of this system (in the $20000-30000 \mathrm{~cm}^{-1}$ spectral range) will be studied. More exact determination of the rovibrational spectrum will also be performed for the $\mathrm{LiH}$ molecule, ${ }^{9}$ as well as for other small molecules with more than two nuclei and/or more than two electrons (e.g., $\mathrm{H}_{3}, \mathrm{LiH}_{2}^{+}$, and $\mathrm{HeH}^{-}$).

In Sec. II we discuss the ECG basis set. Next the derivation of the OO matrix element is presented. In Sec. VII computational implementation is described and some test calculations are shown to validate the correctness of the derived algorithms.

\section{BASIS FUNCTIONS}

Let $n$ denote the number of electrons in the system. The allelectron explicitly correlated Gaussian functions with shifted centers used in this work for expanding the spatial part of the wave function have the following form:

$$
\phi_{k}(\mathbf{r})=\exp \left[-\left(\mathbf{r}-\mathbf{s}_{k}\right)^{T} \underline{\mathbf{A}}_{k}\left(\mathbf{r}-\mathbf{s}_{k}\right)\right],
$$

where $\underline{\mathbf{A}}_{k}$ is a real $3 n \times 3 n$ symmetric matrix of variational exponential parameters and $\mathbf{s}_{k}$ is a $3 n$ vector of the coordinates of the Gaussian centers (shifts). These coordinates are also variational parameters. $T$ denotes the vector/matrix transposition. $\underline{\mathbf{A}}_{k}$ can be written as $\underline{\mathbf{A}}_{k}=\mathbf{A}_{k} \otimes \mathbf{I}_{3}$, where $\mathbf{I}_{3}$ is the $3 \times 3$ unit matrix. To ensure square integrability of $\phi_{k}(\mathbf{r})$, matrix $\mathbf{A}_{k}$ must be positive definite. To ensure this the matrix is represented in the Cholesky factored form as $\mathbf{A}_{k}=\mathbf{L}_{k} \mathbf{L}_{k}{ }^{T}$, where $\mathbf{L}_{k}$ is an $n \times n$, rank $n$, lower triangular matrix. $\phi_{k}(\mathbf{r})$ is square-integrable for the $\mathbf{L}_{k}$ matrix elements being any real numbers.

In the calculation of the wave function expanded in terms of ECGs (1), each Gaussian is transformed with the appropriate permutational symmetry operator. This operator is a sum of operators permuting the electron labels each multiplied by an appropriate linear coefficient. The procedure for generating the permutational symmetry operator was described earlier. ${ }^{10}$ Each labels-permuting operator is represented by a $3 n \times 3 n$ permutation matrix. Let us denote by $\underline{\mathbf{P}}$ the permutation matrix representing a particular $\hat{P}$ permutation operator. Then, acting with $\hat{P}$ on $\phi_{l}$ we get

$$
\begin{aligned}
\hat{P} \exp \left[-\left(\mathbf{r}-\mathbf{s}_{l}\right)^{T} \underline{\mathbf{A}}_{l}\left(\mathbf{r}-\mathbf{s}_{l}\right)\right] & =\exp \left[-\left(\underline{\mathbf{P r}}-\mathbf{s}_{l}\right)^{T} \underline{\mathbf{A}}_{l}\left(\underline{\mathbf{P r}}-\mathbf{s}_{l}\right)\right]=\exp \left[-\left(\underline{\mathbf{P r}}-\underline{\mathbf{P}}^{-1} \mathbf{s}_{l}\right)^{T} \underline{\mathbf{A}}_{l}\left(\underline{\mathbf{P r}}-\underline{\mathbf{P P}}^{-1} \mathbf{s}_{l}\right)\right] \\
& =\exp \left[-\left(\mathbf{r}-\underline{\mathbf{P}}^{-1} \mathbf{s}_{l}\right)^{T} \underline{\mathbf{P}}^{T} \underline{\mathbf{A}} l \underline{\mathbf{P}}\left(\mathbf{r}-\underline{\mathbf{P}}^{-1} \mathbf{s}_{l}\right)\right]=\exp \left[-\left(\mathbf{r}-\tilde{\mathbf{s}}_{l}\right)^{T} \underline{\tilde{\mathbf{A}}}_{l}\left(\mathbf{r}-\tilde{\mathbf{s}}_{l}\right)\right],
\end{aligned}
$$

where $\underline{\tilde{\mathbf{A}}}_{l}=\underline{\mathbf{P}}^{T} \underline{\mathbf{A}}_{l} \underline{\mathbf{P}}$ and $\tilde{\mathbf{s}}_{l}=\underline{\mathbf{P}}^{-1} \mathbf{s}_{l}$. The following notation will help keep expressions more compact:

$$
\left|\tilde{\phi}_{l}\right\rangle=\hat{P}\left|\phi_{l}\right\rangle \text {. }
$$

\section{THE NOTATION}

We start with briefly explaining the notation scheme used throughout this work. We consider a system of $n$ electrons moving in the field of stationary nuclei and $\mathbf{r}$ is a $3 n$-component vector of electron coordinates in an arbitrary Cartesian coordinate system. The notation used is the following:

- $\alpha, \beta$, etc.-lower-case greek letters, and a, b, t, etc.-lower-case latin letters for scalars.

- $\boldsymbol{A}, \boldsymbol{B}, \boldsymbol{X}, \boldsymbol{Y}$, etc.- upper-case latin letters for matrices in the particle space. These are $n \times n$ matrices.

- s, m, etc.-denote an $n$-component column vector in the electron space.

- $\boldsymbol{I}_{3}$ is a $3 \times 3$ identity matrix.

- We define the matrix $\boldsymbol{J}_{i j}$ used in the above expressions as

$$
\boldsymbol{J}_{i j}=\left\{\begin{array}{cc}
\boldsymbol{E}_{i i} & i=j \\
\boldsymbol{E}_{i i}+\boldsymbol{E}_{j j}-\boldsymbol{E}_{i j}-\boldsymbol{E}_{j i} & i \neq j
\end{array}\right\},
$$

where $\boldsymbol{E}_{i j}$ is the $n \times n$ matrix with 1 in the $i j$-th position and 0's elsewhere. Formally this can be written as $\left(\boldsymbol{E}_{i j}\right)_{\beta}^{\alpha}=\delta_{i}^{\alpha} \delta_{j \beta}$. It is easy to see that

$$
\left\{\begin{array}{l}
\boldsymbol{E}_{i j} \cdot \boldsymbol{E}_{i j}=0, \\
\boldsymbol{E}_{i j} \cdot \boldsymbol{E}_{j i}=\boldsymbol{E}_{i i}, \\
\boldsymbol{E}_{i j} \cdot \boldsymbol{E}_{j j}=\boldsymbol{E}_{i j}, \\
\boldsymbol{E}_{j i} \cdot \boldsymbol{E}_{i i}=\boldsymbol{E}_{j i} .
\end{array}\right.
$$

- $\boldsymbol{A} \otimes \boldsymbol{B}-$-stands for the Kronecker product of matrices $\boldsymbol{A}$ and $\boldsymbol{B}$.

- In addition, we also use the following vectors and matrices whose sizes are $3 n$ and $3 n \times 3 n$, respectively, defined with the Kronecker product, $\otimes$, as

$$
\begin{aligned}
& -\mathbf{r}_{i}^{2}=\mathbf{r}^{T} \boldsymbol{J}_{i j} \mathbf{r}, \mathbf{r}_{i j}=\mathbf{r}_{i}-\mathbf{r}_{j}, \text { where } \boldsymbol{r}_{i j} \text { is a } 3 n- \\
& \text { component vector of the distance between electron } \\
& j \text { and electron } i . \\
& -\boldsymbol{A} \otimes \boldsymbol{I}_{3}=\underline{\mathbf{A}} \text { and } \boldsymbol{J}_{i j} \otimes \boldsymbol{I}_{3}=\underline{\mathbf{J}}_{i j}, \text { where } \underline{\mathbf{A}} \text { and } \underline{\mathbf{J}}_{i j} \\
& \text { are } 3 n \times 3 n \text { matrices. }
\end{aligned}
$$

- $\mathbf{A}, \mathbf{B}, \mathbf{X}, \mathbf{Y}$, etc. - are $3 n \times 3 n$ matrices.

- $\overline{\boldsymbol{A}}^{T}, \underline{\mathbf{A}}^{T}$, etc. - stand for the matrix (vector) transpose.

- $\boldsymbol{A}^{-1}, \underline{\mathbf{A}}^{-1}$, etc. - stand for the inverse matrix.

- $|\boldsymbol{A}|,|\underline{\mathbf{A}}|$-vertical bars stand for the determinant of a matrix, where $|\underline{\mathbf{A}}|=|\boldsymbol{A}|^{3}$.

- $\operatorname{Tr}[\boldsymbol{A}], \operatorname{Tr}[\underline{\mathbf{A}}]-$ stand for the trace of matrix $\boldsymbol{A}$ and $\underline{\mathbf{A}}$, respectively, where $\operatorname{Tr}[\underline{\mathbf{A}}]=3 \operatorname{Tr}[\boldsymbol{A}]$. 


\section{SOME AUXILIARY FORMULAS}

There are some simple relations that are used in deriving the expressions for the matrix element of the $\mathrm{OO}$ operator. They include the following:

1.

$$
\int_{-\infty}^{+\infty} \mathrm{d}^{n} x \exp \left[-x^{T} A x+y^{T} x\right]=\frac{\pi^{n / 2}}{|A|^{1 / 2}} \exp \left[\frac{1}{4} y^{T} A^{-1} y\right],
$$

where the integration is over $n$ variables and $x$ is an $n$-component vector of these variables. $y$ is a constant vector, $n \times n$ matrix $A$ is assumed to be symmetric, and its real part is positive definite. Also, here and everywhere below, by the square root one should understand its principal value (i.e., the root whose real part is greater than zero).

2. $F_{0}(t)=\frac{1}{2} \sqrt{\frac{\pi}{t}} \operatorname{Erf}[\sqrt{t}]$.

3. The following symbols are used:

$$
\underline{\mathbf{A}}_{k}+\underline{\tilde{\mathbf{A}}}_{l}=\underline{\mathbf{A}}_{k l} \text {, }
$$

$$
\underline{\mathbf{A}}_{k} \mathbf{s}_{k}=\mathbf{e}_{k} \quad \text { and analogously } \quad \underline{\tilde{\mathbf{A}}}_{l} \tilde{\mathbf{s}}_{l}=\tilde{\mathbf{e}}_{l},
$$

$$
\mathbf{e}_{k}+\tilde{\mathbf{e}}_{l}=\mathbf{e},
$$

$$
\begin{aligned}
-\mathbf{s}_{k}^{T} \underline{\mathbf{A}}_{k} \mathbf{s}_{k}-\tilde{\mathbf{s}}_{l}^{T} \underline{\tilde{\mathbf{A}}}_{l} \tilde{\mathbf{s}}_{l} & =-\eta, \\
\underline{\mathbf{A}}_{k l}^{-1} \mathbf{e} & =\mathbf{s}, \\
\mathbf{e}^{T} \underline{\mathbf{A}}_{k l}^{-1} \mathbf{e}-\eta & =\mathbf{s}^{T} \underline{\mathbf{A}}_{k l} \mathbf{s}-\eta=\gamma, \\
-\eta & =\gamma-\mathbf{s}^{T} \underline{\mathbf{A}}_{k l} \mathbf{s},
\end{aligned}
$$

4. The following useful matrix relations are employed:

- determinant derivative: $\quad d|\mathbf{X}|=|\mathbf{X}| \operatorname{Tr}\left[\mathbf{X}^{-1} d \mathbf{X}\right]$,

- $d|\mathbf{X}|^{-3 / 2}=-\frac{3}{2}|\mathbf{X}|^{-5 / 2}|\mathbf{X}| \operatorname{Tr}\left[\mathbf{X}^{-1} d \mathbf{X}\right]=-\frac{3}{2}|\mathbf{X}|^{-3 / 2} \operatorname{Tr}\left[\mathbf{X}^{-1} d \mathbf{X}\right]$,

- differential of a matrix inverse: $\quad d \mathbf{X}^{-1}=-\mathbf{X}^{-1}(d \mathbf{X}) \mathbf{X}^{-1}$.

5. By $\nabla_{\mathbf{r}}=\left[\partial_{x}, \partial_{y}, \partial_{z}\right]$ we denote the gradient with respect to the vector of the coordinates, $\mathbf{r}$, and we have

$$
\nabla_{\mathbf{r}}^{\alpha} \phi \equiv \nabla^{\alpha} \phi \equiv \partial^{\alpha} \phi \quad \text { and } \quad \nabla_{\mathbf{r}_{\alpha}} \phi \equiv \nabla_{\alpha} \phi \equiv \partial_{\alpha} \phi .
$$

6. The following first-order derivatives of function $\phi_{k}=\exp \left[-\left(\mathbf{r}-\mathbf{s}_{k}\right)^{T} \underline{\mathbf{A}}_{k}\left(\mathbf{r}-\mathbf{s}_{k}\right)\right]$ are used:

$$
\begin{aligned}
& \nabla^{\alpha} \phi_{k} \equiv \partial^{\alpha} \phi_{k}=-2\left[\left(\mathbf{r}-\mathbf{s}_{k}\right)^{T} \underline{\mathbf{A}}_{k}\right]^{\alpha} \phi_{k}, \\
& \nabla_{\alpha} \phi_{k} \equiv \partial_{\alpha} \phi_{k}=-2\left[\underline{\mathbf{A}}_{k}\left(\mathbf{r}-\mathbf{s}_{k}\right)\right]_{\alpha} \phi_{k} .
\end{aligned}
$$

With that we have the following useful relations:

$$
\begin{aligned}
\partial^{\alpha} \partial_{\beta} \phi_{k}= & \left\{-2\left(\underline{\mathbf{A}}_{k}\right)_{\beta}^{\alpha}+4\left[\left(\mathbf{r}-\mathbf{s}_{k}\right)^{T} \underline{\mathbf{A}}_{k}\right]^{\alpha}\left[\underline{\mathbf{A}}_{k}\left(\mathbf{r}-\mathbf{s}_{k}\right)\right]_{\beta}\right\} \phi_{k} \\
\partial_{\alpha} \partial_{\beta} \phi_{k}= & \left\{-2\left(\underline{\mathbf{A}}_{k}\right)_{\alpha \beta}+4\left[\underline{\mathbf{A}}_{k}\left(\mathbf{r}-\mathbf{s}_{k}\right)\right]_{\alpha}\left[\underline{\mathbf{A}}_{k}\left(\mathbf{r}-\mathbf{s}_{k}\right)\right]_{\beta}\right\} \phi_{k} \\
\partial^{\rho} \partial_{\beta} \partial_{\alpha} \phi_{k}= & \left\{4\left(\underline{\mathbf{A}}_{k}\right)_{\beta}^{\rho}\left[\underline{\mathbf{A}}_{k}\left(\mathbf{r}-\mathbf{s}_{k}\right)\right]_{\alpha}+4\left(\underline{\mathbf{A}}_{k}\right)_{\alpha}^{\rho}\left[\underline{\mathbf{A}}_{k}\left(\mathbf{r}-\mathbf{s}_{k}\right)\right]_{\beta}+4\left[\left(\mathbf{r}-\mathbf{s}_{k}\right)^{T} \underline{\mathbf{A}}_{k}\right]^{\rho}\left(\underline{\mathbf{A}}_{k}\right)_{\beta \alpha}+\right. \\
& \left.-8\left[\left(\mathbf{r}-\mathbf{s}_{k}\right)^{T} \underline{\mathbf{A}}_{k}\right]^{\rho}\left[\underline{\mathbf{A}}_{k}\left(\mathbf{r}-\mathbf{s}_{k}\right)\right]_{\beta}\left[\underline{\mathbf{A}}_{k}\left(\mathbf{r}-\mathbf{s}_{k}\right)\right]_{\alpha}\right\} \phi_{k} \\
(\underline{\mathbf{D}} \nabla)_{\beta} \phi_{k}= & \underline{\mathbf{D}}_{\beta}{ }^{\alpha} \partial_{\alpha} \phi_{k}=-2\left[\underline{\mathbf{D}} \underline{\mathbf{A}}_{k}\left(\mathbf{r}-\mathbf{s}_{k}\right)\right]_{\beta} \phi_{k} \\
\left(\nabla^{T} \underline{\mathbf{D}}\right)^{\beta} \phi_{k}= & \underline{\mathbf{D}}_{\alpha}{ }^{\beta} \partial^{\alpha} \phi_{k}=-2\left[\left(\mathbf{r}-\mathbf{s}_{k}\right)^{T} \underline{\mathbf{A}}_{k} \underline{\mathbf{D}}\right]^{\beta} \phi_{k} \\
\nabla^{T} \underline{\mathbf{D}} \nabla \phi_{k}= & \left\{-6 \operatorname{Tr}\left[A_{k} D\right]+4\left(\mathbf{r}-\mathbf{s}_{k}\right)^{T} \underline{\mathbf{A}}_{k} \underline{\mathbf{D}} \underline{\mathbf{A}}_{k}\left(\mathbf{r}-\mathbf{s}_{k}\right)\right\} \phi_{k}
\end{aligned}
$$

where $\underline{\mathbf{D}}$ is an arbitrary $3 n \times 3 n$ matrix. 
7. The following derivatives are also used in this work (for an arbitrary square matrix $\mathbf{X}$ dependent on parameters $\alpha$ and $\beta$ : $\mathbf{X}=\mathbf{X}(\alpha, \beta))$ :

$$
\begin{aligned}
& \partial_{\alpha}\left\{|\mathbf{X}|^{-3 / 2} \exp \left[\mathbf{e}^{T} \mathbf{X}^{-1} \mathbf{e}\right]\right\}=-|\mathbf{X}|^{-3 / 2} \exp \left[\mathbf{e}^{T} \mathbf{X}^{-1} \mathbf{e}\right]\left\{\frac{3}{2} \operatorname{Tr}\left[\mathbf{X}^{-1} \partial_{\alpha} \mathbf{X}\right]+\left[\mathbf{e}^{T} \mathbf{X}^{-1}\left(\partial_{\alpha} \mathbf{X}\right) \mathbf{X}^{-1} \mathbf{e}\right]\right\}, \\
& \partial_{\beta} \partial_{\alpha}\left\{|\mathbf{X}|^{-3 / 2} \exp \left[\mathbf{e}^{T} \mathbf{X}^{-1} \mathbf{e}\right]\right\}=|\mathbf{X}|^{-3 / / 2} \exp \left[\mathbf{e}^{T} \mathbf{X}^{-1} \mathbf{e}\right]\left(\frac{3}{2}\left(\mathbf{e}^{T} \mathbf{X}^{-1}\left(\partial_{\beta} \mathbf{X}\right) \mathbf{X}^{-1} \mathbf{e}\right) \operatorname{Tr}\left[\mathbf{X}^{-1} \partial_{\alpha} \mathbf{X}\right]+\left(\mathbf{e}^{T} \mathbf{X}^{-1}\left(\partial_{\beta} \mathbf{X}\right) \mathbf{X}^{-1} \mathbf{e}\right)\right. \\
& \times\left(\mathbf{e}^{T} \mathbf{X}^{-1}\left(\partial_{\alpha} \mathbf{X}\right) \mathbf{X}^{-1} \mathbf{e}\right)+\frac{9}{4} \operatorname{Tr}\left[\mathbf{X}^{-1} \partial_{\beta} \mathbf{X}\right] \operatorname{Tr}\left[\mathbf{X}^{-1} \partial_{\alpha} \mathbf{X}\right]+\frac{3}{2} \operatorname{Tr}\left[\mathbf{X}^{-1}\left(\partial_{\beta} \mathbf{X}\right) \mathbf{X}^{-1} \partial_{\alpha} \mathbf{X}\right. \\
&\left.-\mathbf{X}^{-1} \partial_{\beta} \partial_{\alpha} \mathbf{X}\right]+\frac{3}{2} \operatorname{Tr}\left[\mathbf{X}^{-1} \partial_{\beta} \mathbf{X}\right]\left(\mathbf{e}^{T} \mathbf{X}^{-1}\left(\partial_{\alpha} \mathbf{X}\right) \mathbf{X}^{-1} \mathbf{e}\right)+\left(\mathbf{e}^{T} \mathbf{X}^{-1}\left(\partial_{\beta} \mathbf{X}\right) \mathbf{X}^{-1}\left(\partial_{\alpha} \mathbf{X}\right) \mathbf{X}^{-1} \mathbf{e}\right) \\
&\left.-\left(\mathbf{e}^{T} \mathbf{X}^{-1}\left(\partial_{\beta} \partial_{\alpha} \mathbf{X}\right) \mathbf{X}^{-1} \mathbf{e}\right)+\left(\mathbf{e}^{T} \mathbf{X}^{-1}\left(\partial_{\alpha} \mathbf{X}\right) \mathbf{X}^{-1}\left(\partial_{\beta} \mathbf{X}\right) \mathbf{X}^{-1} \mathbf{e}\right)\right) .
\end{aligned}
$$

8. With the above-defined quantities it can be now shown that the operators $\nabla_{i}^{T} \cdot \nabla_{j}$ and $\mathbf{r}_{i j}^{T} \nabla_{j}$ can be expressed as

$$
\begin{gathered}
\nabla_{i}^{T} \cdot \nabla_{j}=\nabla^{T} \underline{\mathbf{E}}_{i j} \nabla, \\
\mathbf{r}_{i j}^{T} \cdot \nabla_{j}=\left(\mathbf{r}_{i}-\mathbf{r}_{j}\right)^{T} \cdot \nabla_{j}=\mathbf{r}^{T}\left(\underline{\mathbf{E}}_{i j}-\underline{\mathbf{E}}_{j j}\right) \nabla_{j} \\
=\mathbf{r}^{T}\left(\underline{\mathbf{E}}_{i j}-\underline{\mathbf{E}}_{j j}\right) \underline{\mathbf{E}}_{j j} \nabla .
\end{gathered}
$$

9. Transformations involving determinants can be handled using the following theorem:

Theorem. 1 (on the inverse matrix and the determinant) (Ref. 11). If

- $\mathbf{G}$ and $\mathbf{G}+\mathbf{H}$ are non-singular matrices,

- $\operatorname{rank} \mathbf{H}=r>0$,

- $\mathbf{H}=\mathbf{H}_{1}+\mathbf{H}_{2}+\cdots+\mathbf{H}_{r}$, where rank $\mathbf{H}_{k}=1$, $1 \leq k \leq r$, and

- $\mathbf{C}_{k+1}=\mathbf{G}+\mathbf{H}_{1}+\cdots+\mathbf{H}_{k}$ is non-singular for $k=1, \ldots, r\left(\mathbf{C}_{1}=\mathbf{G}\right)$,

then

(a) $\mathbf{C}_{k+1}^{-1}=\mathbf{C}_{k}^{-1}-v_{k} \mathbf{C}_{k}^{-1} \mathbf{H}_{k} \mathbf{C}_{k}^{-1}$, where $v_{k}^{-1}=1+\operatorname{Tr} \mathbf{C}_{k}^{-1} \mathbf{H}$ $1 \leq k \leq r$

(b) $|\mathbf{G}+\mathbf{H}|=\left(v_{1} v_{2} \ldots v_{r}\right)^{-1}|\mathbf{G}|$.

Using the above, we can express the determinants $\left|\mathbf{I}_{n}+a \mathbf{H}_{1}\right|$ and $\left|\mathbf{I}_{n}+a \mathbf{H}_{1}+b \mathbf{H}_{2}\right|$ as sums. To write the expressions for the sums we need the following:

$$
\begin{aligned}
\mathbf{C}_{1}^{-1} & =\mathbf{I}_{n}, \\
v_{1}^{-1} & =1+a \operatorname{Tr}\left[\mathbf{H}_{1}\right], \\
\mathbf{C}_{2}^{-1} & =\left(1+a \operatorname{Tr}\left[\mathbf{H}_{1}\right]\right)^{-1} \mathbf{H}_{1}, \\
v_{2}^{-1} & =1+b \operatorname{Tr}\left[\mathbf{H}_{2}\right]-\left(1+a \operatorname{Tr}\left[\mathbf{H}_{1}\right]\right)^{-1} a b \operatorname{Tr}\left[\mathbf{H}_{1} \mathbf{H}_{2}\right] .
\end{aligned}
$$

This leads to

$$
\left|\mathbf{I}_{n}+a \mathbf{H}_{1}\right|=1+a \operatorname{Tr}\left[\mathbf{H}_{1}\right]
$$

and

$$
\begin{aligned}
\left|\mathbf{I}_{n}+a \mathbf{H}_{1}+b \mathbf{H}_{2}\right|= & 1+a \operatorname{Tr}\left[\mathbf{H}_{1}\right]+b \operatorname{Tr}\left[\mathbf{H}_{2}\right] \\
& +a b\left(\operatorname{Tr}\left[\mathbf{H}_{1}\right] \operatorname{Tr}\left[\mathbf{H}_{2}\right]-\operatorname{Tr}\left[\mathbf{H}_{1} \mathbf{H}_{2}\right]\right) .
\end{aligned}
$$

10. The inverse of a sum of matrices

- If $\boldsymbol{A}$ is an arbitrary nonsingular square matrix and $\boldsymbol{B}$ is a nonsingular matrix with rank one, then there exist non-zero vectors $\mathbf{u}$ and $\mathbf{v}$ such that $\boldsymbol{B}=\mathbf{u v}^{T}$ and the inverse of the sum of $\boldsymbol{A}$ and $\boldsymbol{B}$ has the following form: ${ }^{11}$

$$
(\boldsymbol{A}+\boldsymbol{B})^{-1}=\boldsymbol{A}^{-1}-\frac{\boldsymbol{A}^{-1} \boldsymbol{B} \boldsymbol{A}^{-1}}{1+\operatorname{Tr}\left[\boldsymbol{B} \boldsymbol{A}^{-1}\right]} .
$$

- The inverse of the sum of two Kronecker products, $\underline{\mathbf{W}}=\boldsymbol{A} \otimes \boldsymbol{G}+\boldsymbol{B} \otimes \boldsymbol{E}$, where $\boldsymbol{B}$ and $\boldsymbol{E}$ are matrices of rank one, and $\boldsymbol{A}, \boldsymbol{G}$, and $\boldsymbol{W}$ are non-singular, is

$$
\begin{aligned}
\underline{\mathbf{W}}^{-1} & =(\boldsymbol{A} \otimes \boldsymbol{G}+\boldsymbol{B} \otimes \boldsymbol{E})^{-1} \\
& =\boldsymbol{A}^{-1} \otimes \boldsymbol{G}^{-1}-\frac{1}{1+\mathrm{ga}} \boldsymbol{A}^{-1} \boldsymbol{B} \boldsymbol{A}^{-1} \otimes \boldsymbol{G}^{-1} \boldsymbol{E} \boldsymbol{G}^{-1},
\end{aligned}
$$

where $\mathrm{g}=\operatorname{Tr}\left[\boldsymbol{E} \boldsymbol{G}^{-1}\right] \quad$ and $\quad \mathrm{a}=\operatorname{Tr}\left[\boldsymbol{B} \boldsymbol{A}^{-1}\right]$.

If $\boldsymbol{G}=\boldsymbol{E}=\boldsymbol{I}_{3}$, then

$$
\begin{aligned}
\underline{\mathbf{W}}^{-1} & =\left(\boldsymbol{A} \otimes \boldsymbol{I}_{3}+\boldsymbol{B} \otimes \boldsymbol{I}_{3}\right)^{-1} \\
& =\boldsymbol{A}^{-1} \otimes \boldsymbol{I}_{3}^{-1}-\frac{1}{1+\mathrm{a}} \boldsymbol{A}^{-1} \boldsymbol{B} \boldsymbol{A}^{-1} \otimes \boldsymbol{I}_{3}^{-1},
\end{aligned}
$$

because $\mathrm{g}=\operatorname{Tr}\left[\boldsymbol{I}_{3} \cdot \boldsymbol{I}_{3}^{-1}\right]=1 \quad$ and $\quad \mathrm{a}=\operatorname{Tr}\left[\boldsymbol{B} \boldsymbol{A}^{-1}\right]$.

\section{ORBIT-ORBIT MATRIX ELEMENT}

The general form of the operator representing the orbitorbit magnetic interaction for a system of $N$ particles with charges $Q_{i}$ and masses $M_{i}$ is

$\hat{H}_{\mathrm{OO}}=-\frac{1}{2} \sum_{i=1}^{N} \sum_{j} \frac{Q_{i} Q_{j}}{M_{i} M_{j}}\left[\frac{1}{r_{i j}}\left(\nabla_{i}^{T} \cdot \nabla_{j}\right)+\frac{\mathbf{r}_{i j}^{T}}{r_{i j}^{3}}\left(\mathbf{r}_{i j}^{T} \cdot \nabla_{i}\right) \cdot \nabla_{j}\right]$,

where $r_{i j}$ is the distance between particles $i$ and $j$, and $\mathbf{r}_{i j}$ is the corresponding distance vector. Using the relation

$$
\frac{\mathbf{r}^{T}\left(\underline{\mathbf{E}}_{j i}-\underline{\mathbf{E}}_{i i}\right)}{r_{i j}^{3}}=\left(\nabla^{T} \underline{\mathbf{E}}_{j i} \frac{1}{r_{i j}}\right)
$$

and the matrix notation described earlier, the $k l$ matrix element of the orbit-orbit interaction operator is 


$$
\begin{aligned}
\left\langle\phi_{k}\left|\hat{H}_{\mathrm{OO}}(\mathbf{r})\right| \tilde{\phi}_{l}\right\rangle & =-\frac{1}{2} \sum_{i=1}^{N} \sum_{j>i}^{N} \frac{Q_{i} Q_{j}}{M_{i} M_{j}}\left\{\left\langle\phi_{k}\left|\frac{1}{r_{i j}}\left(\nabla^{T} \underline{\mathbf{E}}_{i j} \nabla\right)+\left(\mathbf{r}^{T}\left(\underline{\mathbf{E}}_{i j}-\underline{\mathbf{E}}_{j j}\right)\right)^{\alpha}\left(\nabla^{T} \underline{\mathbf{E}}_{j i} \frac{1}{r_{i j}}\right)^{\beta}\left(\underline{\mathbf{E}}_{i i} \nabla\right)_{\beta}\left(\underline{\mathbf{E}}_{j j} \nabla\right)_{\alpha}\right| \tilde{\phi}_{l}\right\rangle\right\} \\
& =-\frac{1}{2} \sum_{i=1}^{N} \sum_{j>i}^{N} \frac{Q_{i} Q_{j}}{M_{i} M_{j}}\left\{\left\langle\phi_{k}\left|\frac{1}{r_{i j}}\left(\nabla^{T} \underline{\mathbf{E}}_{i j} \nabla\right)\right| \phi_{l}\right\rangle+\left\langle\phi_{k}\left|\left(\mathbf{r}^{T}\left(\underline{\mathbf{E}}_{i j}-\underline{\mathbf{E}}_{j j}\right)\right)^{\alpha}\left(\nabla^{T} \underline{\mathbf{E}}_{j i} \frac{1}{r_{i j}}\right)^{\beta}\left(\underline{\mathbf{E}}_{i i} \nabla\right)_{\beta}\left(\underline{\mathbf{E}}_{j j} \nabla\right)_{\alpha}\right| \tilde{\phi}_{l}\right\rangle\right\} .
\end{aligned}
$$

We now derive formulas for the two integrals in the above equation.

\section{A. Integral $\left\langle\phi_{k}\left|\frac{1}{r_{i j}} \nabla^{T} \underline{E}_{i j} \nabla\right| \tilde{\phi}_{I}\right\rangle$}

First we apply the operator $\nabla^{T} \underline{\mathbf{E}}_{i j} \nabla$ to $\phi_{l}$. Using relation (21) we get

$$
\left\langle\phi_{k}\left|\frac{1}{r_{i j}}\left(\nabla^{T} \underline{\mathbf{E}}_{i j} \nabla\right)\right| \tilde{\phi}_{l}\right\rangle=-6 \operatorname{Tr}\left[\boldsymbol{A}_{l} \boldsymbol{E}_{i j}\right]\left\langle\phi_{k}\left|\frac{1}{r_{i j}}\right| \tilde{\phi}_{l}\right\rangle+4\left\langle\phi_{k}\left|\frac{1}{r_{i j}}\left(\mathbf{r}-\mathbf{s}_{l}\right)^{T} \underline{\mathbf{A}}_{l} \underline{\mathbf{D}} \underline{\mathbf{A}}_{l}\left(\mathbf{r}-\mathbf{s}_{l}\right)\right| \tilde{\phi}_{l}\right\rangle .
$$

The integrals which appear in the above expression were derived before in the work concerning the Darwin relativistic correction. ${ }^{8}$

\section{B. Integral $\left\langle\phi_{k}\left|\left(\mathbf{r}^{T}\left(\underline{E}_{i j}-\underline{E}_{j j}\right)\right)^{\alpha}\left(\nabla^{T} \underline{E}_{j i} \frac{1}{r_{i j}}\right)^{\beta}\left(\underline{E}_{i i} \nabla\right)_{\beta}\left(\underline{E}_{j j} \nabla\right)_{\alpha}\right| \tilde{\phi}_{I}\right\rangle$}

To simplify this integral, we use the following general integral:

$$
\left\langle\phi_{k}\left|\left(\mathbf{r}^{T} \underline{\mathbf{C}}\right)^{\alpha}\left(\nabla^{T} \underline{\mathbf{D}} \frac{1}{r_{i j}}\right)^{\beta}(\underline{\mathbf{F}} \nabla)_{\beta}(\underline{\mathbf{G}} \nabla)_{\alpha}\right| \tilde{\phi}_{l}\right\rangle,
$$

where

$$
\underline{\mathbf{C}}=\left(\underline{\mathbf{E}}_{i j}-\underline{\mathbf{E}}_{j j}\right), \underline{\mathbf{D}}=\underline{\mathbf{E}}_{j i}, \underline{\mathbf{F}}=\underline{\mathbf{E}}_{i i}, \text { and } \underline{\mathbf{G}}=\underline{\mathbf{E}}_{j j} .
$$

Now rewrite this integral in the following way:

$$
\left\langle\phi_{k}\left|\left(\mathbf{r}^{T} \underline{\mathbf{C}}\right)^{\alpha}\left(\nabla^{T} \underline{\mathbf{D}} \frac{1}{r_{i j}}\right)^{\beta}(\underline{\mathbf{F}} \nabla)_{\beta}(\underline{\mathbf{G}} \nabla)_{\alpha}\right| \tilde{\phi}_{l}\right\rangle=-\int d r^{3} \frac{1}{r_{i j}}\left(\nabla^{T} \underline{\mathbf{D}}\right)^{\beta}\left\{\phi_{k}\left(\mathbf{r}^{T} \underline{\mathbf{C}}\right)^{\alpha}(\underline{\mathbf{F}} \nabla)_{\beta}(\underline{\mathbf{G}} \nabla)_{\alpha} \tilde{\phi}_{l}\right\} .
$$

The integral can be split into three terms,

$$
\begin{aligned}
-\int d r^{3} \frac{1}{r_{i j}}\left(\nabla^{T} \underline{\mathbf{D}}\right)^{\beta}\left\{\phi_{k}\left(\mathbf{r}^{T} \underline{\mathbf{C}}\right)^{\alpha}(\underline{\mathbf{F}} \nabla)_{\beta}(\underline{\mathbf{G}} \nabla)_{\alpha} \tilde{\phi}_{l}\right\}= & -\int d r^{3} \frac{1}{r_{i j}}\left\{\left(\nabla^{T} \underline{\mathbf{D}}\right)^{\beta} \phi_{k}\right\}\left(\mathbf{r}^{T} \underline{\mathbf{C}} \underline{\mathbf{G}} \nabla\right)(\underline{\mathbf{F}} \nabla)_{\beta} \tilde{\phi}_{l} \quad \equiv \operatorname{term} 1 \\
& -\int d r^{3} \frac{1}{r_{i j}} \phi_{k}\left\{\left(\nabla^{T} \underline{\mathbf{D}}\right)^{\beta}\left(\mathbf{r}^{T} \underline{\mathbf{C}}\right)^{\alpha}\right\}(\underline{\mathbf{F}} \nabla)_{\beta}(\underline{\mathbf{G}} \nabla)_{\alpha} \tilde{\phi}_{l} \equiv \operatorname{term} 2 \\
& -\int d r^{3} \frac{1}{r_{i j}} \phi_{k}\left(\mathbf{r}^{T} \underline{\mathbf{C}}\right)^{\alpha}\left\{\left(\nabla^{T} \underline{\mathbf{D}}\right)^{\beta}(\underline{\mathbf{F}} \nabla)_{\beta}(\underline{\mathbf{G}} \nabla)_{\alpha} \tilde{\phi}_{l}\right\} \equiv \operatorname{term} 3 .
\end{aligned}
$$

We now evaluate each term separately.

\section{Term $1-\int d r^{3} \frac{1}{r_{i j}}\left\{\left(\nabla^{T} \underline{D}\right)^{\beta} \phi_{k}\right\}\left(r^{T} \underline{C} \nabla\right)(\underline{F} \nabla)_{\beta} \tilde{\phi}_{l}$, where $\underline{C} \underline{G}=\underline{C}$.}

The integrand is now separated into two terms which are evaluated separately as follows:

$$
\begin{aligned}
& \text { - }\left(\nabla^{T} \underline{\mathbf{D}}\right)^{\beta} \phi_{k} \stackrel{(20)}{=}-2\left[\left(\mathbf{r}-\mathbf{s}_{k}\right)^{T} \underline{\mathbf{A}_{k}} \underline{\mathbf{D}}\right]^{\beta} \phi_{k}, \\
& \text { - }\left(\mathbf{r}^{T} \underline{\mathbf{C}} \nabla\right)(\underline{\mathbf{F}} \nabla)_{\beta} \tilde{\phi}_{l}=\left(\mathbf{r}^{T} \underline{\mathbf{C}}\right)^{\alpha} \underline{\mathbf{F}}_{\beta}{ }^{\rho} \partial_{\alpha} \partial_{\rho} \tilde{\phi}_{l} \stackrel{(17)}{=}\left(\mathbf{r}^{T} \underline{\mathbf{C}}\right)^{\alpha} \underline{\mathbf{F}}_{\beta}{ }^{\rho}\left\{-2\left(\underline{\tilde{\mathbf{A}}}_{l}\right)_{\alpha \rho}+4\left[\underline{\tilde{\mathbf{A}}}_{l}\left(\mathbf{r}-\tilde{\mathbf{s}}_{l}\right)\right]_{\alpha}\left[\underline{\tilde{\mathbf{A}}}_{l}\left(\mathbf{r}-\tilde{\mathbf{s}}_{l}\right)\right]_{\rho}\right\} \tilde{\phi}_{l} \\
& =\left\{4\left(\mathbf{r}-\mathbf{s}_{k}\right)^{T} \underline{\mathbf{A}}_{k} \underline{\mathbf{D}} \underline{\mathbf{F}} \underline{\tilde{\mathbf{A}}}_{l} \underline{\mathbf{C}}^{T} \mathbf{r}-8 \mathbf{r}^{T} \underline{\mathbf{C}}_{\underline{\tilde{A}}_{l}}\left(\mathbf{r}-\tilde{\mathbf{s}}_{l}\right)\left(\mathbf{r}-\mathbf{s}_{k}\right)^{T} \underline{\mathbf{A}}_{k} \underline{\mathbf{D}} \underline{\mathbf{F}}_{l}^{\tilde{\mathbf{A}}_{l}}\left(\mathbf{r}-\tilde{\mathbf{s}}_{l}\right)\right\} \phi_{k} \tilde{\phi}_{l} .
\end{aligned}
$$

With that and using (31) the integral becomes 


$$
\begin{aligned}
\int d r^{3} \frac{1}{r_{i j}}\left\{\left(\nabla^{T} \underline{\mathbf{E}}_{i j}\right)^{\beta} \phi_{k}\right\}\left(\mathbf{r}^{T}\left(\underline{\mathbf{E}}_{i j}-\underline{\mathbf{E}}_{j j}\right) \nabla\right)\left(\underline{\mathbf{E}}_{i i} \nabla\right)_{\beta} \tilde{\phi}_{l}= & -8\left\langle\phi_{k}\left|\frac{1}{r_{i j}}\left(\mathbf{r}-\mathbf{s}_{k}\right)^{T} \underline{\mathbf{A}}_{k} \underline{\mathbf{E}}_{j i} \underline{\tilde{\mathbf{A}}}_{l}\left(\mathbf{r}-\tilde{\mathbf{s}}_{l}\right) \mathbf{r}^{T}\left(\underline{\mathbf{E}}_{i j}-\underline{\mathbf{E}}_{j j}\right) \underline{\tilde{\mathbf{A}}}_{l}\left(\mathbf{r}-\tilde{\mathbf{s}}_{l}\right)\right| \tilde{\phi}_{l}\right\rangle \\
& +4 \operatorname{Tr}\left[\underline{\tilde{\mathbf{A}}}_{l} \underline{\mathbf{E}}_{i i}\right]\left\langle\phi_{k}\left|\frac{1}{r_{i j}}\left(\mathbf{r}-\mathbf{s}_{k}\right)^{T} \underline{\mathbf{A}}_{k}\left(\underline{\mathbf{E}}_{j i}-\underline{\mathbf{E}}_{j j}\right) \mathbf{r}\right| \tilde{\phi}_{l}\right\rangle .
\end{aligned}
$$

2. Term $2 \int d^{3} r \phi_{k} \frac{1}{r_{i j}}\left\{\left(\nabla^{T} \underline{D}\right)^{\beta}\left(r^{T} \underline{C}\right)^{\alpha}\right\}(\underline{F} \nabla)_{\beta}(\underline{G} \nabla)_{\alpha} \tilde{\phi}_{I}$

We first transform the expression in the integral in the following way:

$$
\begin{gathered}
\left(\nabla^{T} \underline{\mathbf{D}}\right)^{\beta}\left(\mathbf{r}^{T} \underline{\mathbf{C}}\right)^{\alpha}=\underline{\mathbf{D}}_{\gamma}^{\beta} \partial^{\gamma} \mathbf{r}^{\rho} \underline{\mathbf{C}}_{\rho}{ }^{\alpha}=\underline{\mathbf{D}}_{\gamma}^{\beta} \underline{\mathbf{C}}^{\gamma \alpha}, \\
\left\{\left(\nabla^{T} \underline{\mathbf{D}}\right)^{\beta}\left(\mathbf{r}^{T} \underline{\mathbf{C}}\right)^{\alpha}\right\}(\underline{\mathbf{F}} \nabla)_{\beta}(\underline{\mathbf{G}} \nabla)_{\alpha} \tilde{\phi}_{l}=\underline{\mathbf{D}}_{\gamma}^{\beta} \underline{\mathbf{C}}^{\gamma \alpha}(\underline{\mathbf{F}} \nabla)_{\beta}(\underline{\mathbf{G}} \nabla)_{\alpha} \tilde{\phi}_{l}=\left(\nabla^{T} \underline{\mathbf{G}}^{T} \underline{\mathbf{C}}^{T} \underline{\mathbf{D}} \underline{\mathbf{F}} \nabla\right) \tilde{\phi}_{l},
\end{gathered}
$$

and we obtain an expression analogical to the Darwin term ${ }^{8}$ using (31),

$$
\left\langle\phi_{k}\left|\frac{1}{r_{i j}}\left\{\left(\nabla^{T} \underline{\mathbf{E}}_{j i}\right)^{\beta}\left(\mathbf{r}^{T}\left(\underline{\mathbf{E}}_{i j}-\underline{\mathbf{E}}_{j j}\right)\right)^{\alpha}\right\}\left(\underline{\mathbf{E}}_{i i} \nabla\right)_{\beta}\left(\underline{\mathbf{E}}_{j j} \nabla\right)_{\alpha}\right| \tilde{\phi}_{l}\right\rangle=-\left\langle\phi_{k}\left|\frac{1}{r_{i j}}\left(\nabla^{T} \underline{\mathbf{E}}_{j i} \nabla\right)\right| \tilde{\phi}_{l}\right\rangle .
$$

\section{Term $3 \int d^{3} \phi_{k} \frac{1}{r_{i j}}\left(r^{T} \underline{C}\right)^{\alpha}\left\{\left(\nabla^{T} \underline{D}\right)^{\beta}(\underline{F} \nabla)_{\beta}(\underline{G} \nabla)_{\alpha} \tilde{\phi}_{i}\right\}$}

First we make a few simple transformations

$$
\begin{aligned}
& \text { - }\left(\mathbf{r}^{T} \underline{\mathbf{C}}\right)^{\alpha}\left\{\left(\nabla^{T} \underline{\mathbf{D}}\right)^{\beta}(\underline{\mathbf{F}} \nabla)_{\beta}(\underline{\mathbf{G}} \nabla)_{\alpha} \tilde{\phi}_{l}\right\}=\left(\mathbf{r}^{T} \underline{\mathbf{C}}\right)^{\xi}(\underline{\mathbf{D}})_{\gamma}{ }^{\eta} \partial^{\gamma} \partial_{\eta} \partial_{\xi} \tilde{\phi}_{l} \\
& \stackrel{(18)}{=}\left(\mathbf{r}^{T} \underline{\mathbf{C}}\right)^{\xi}(\underline{\mathbf{D}})_{\gamma}{ }^{\eta}\left\{4\left(\underline{\tilde{\mathbf{A}}}_{l}\right)_{\eta}^{\gamma}\left[\underline{\tilde{\mathbf{A}}}_{l}\left(\mathbf{r}-\tilde{\mathbf{s}}_{l}\right)\right]_{\xi}+4\left(\underline{\tilde{\mathbf{A}}}_{l}\right)_{\xi}^{\gamma}\left[\underline{\tilde{\mathbf{A}}}_{l}\left(\mathbf{r}-\tilde{\mathbf{s}}_{l}\right)\right]_{\eta}+4\left[\left(\mathbf{r}-\tilde{\mathbf{s}}_{l}\right)^{T} \underline{\tilde{\mathbf{A}}}_{l}\right]^{\gamma}\left(\underline{\tilde{\mathbf{A}}}_{l}\right)_{\eta \xi}+\right. \\
& \left.-8\left[\left(\mathbf{r}-\tilde{\mathbf{s}}_{l}\right)^{T} \underline{\tilde{\mathbf{A}}}_{l}\right]^{\gamma}\left[\underline{\tilde{\mathbf{A}}}_{l}\left(\mathbf{r}-\tilde{\mathbf{s}}_{l}\right)\right]_{\eta}\left[\underline{\tilde{\mathbf{A}}}_{l}\left(\mathbf{r}-\tilde{\mathbf{s}}_{l}\right)\right]_{\xi}\right\} \tilde{\phi}_{l} \\
& =\left\{4 \operatorname{Tr}\left[\underline{\mathbf{D}} \underline{\tilde{\mathbf{A}}}_{l}\right] \mathbf{r}^{T} \underline{\mathbf{C}} \underline{\tilde{\mathbf{A}}}_{l}\left(\mathbf{r}-\tilde{\mathbf{s}}_{l}\right)+4 \mathbf{r}^{T} \underline{\mathbf{C}} \underline{\tilde{\mathbf{A}}}_{l} \underline{\mathbf{D}} \underline{\tilde{\mathbf{A}}}_{l}\left(\mathbf{r}-\tilde{\mathbf{s}}_{l}\right)+4\left(\mathbf{r}-\tilde{\mathbf{s}}_{l}\right)^{T} \underline{\tilde{\mathbf{A}}}_{l} \underline{\mathbf{D}} \underline{\tilde{\mathbf{A}}}_{l} \underline{\mathbf{C}}^{T} \mathbf{r}+\right. \\
& \left.-8\left(\mathbf{r}-\tilde{\mathbf{s}}_{l}\right)^{T} \underline{\tilde{\mathbf{A}}}_{l} \underline{\mathbf{D}} \underline{\tilde{\mathbf{A}}}_{l}\left(\mathbf{r}-\tilde{\mathbf{s}}_{l}\right) \mathbf{r}^{T} \underline{\mathbf{C}}_{\tilde{\mathbf{A}}_{l}}\left(\mathbf{r}-\tilde{\mathbf{s}}_{l}\right)\right\} \tilde{\phi}_{l} \text {. }
\end{aligned}
$$

With that term 3 can be expressed as a sum of the following integrals:

$$
\begin{gathered}
\int d^{3} \phi_{k} \frac{1}{r_{i j}}\left(\mathbf{r}^{T}\left(\underline{\mathbf{E}}_{i j}-\underline{\mathbf{E}}_{j j}\right)\right)^{\alpha}\left\{\left(\nabla^{T} \underline{\mathbf{E}}_{j i}\right)^{\beta}\left(\underline{\mathbf{E}}_{i i} \nabla\right)_{\beta}\left(\underline{\mathbf{E}}_{j j} \nabla\right)_{\alpha} \tilde{\phi}_{l}\right\}= \\
=4 \operatorname{Tr}\left[\underline{\mathbf{E}}_{j i} \underline{\tilde{\mathbf{A}}}_{l}\right]\left\langle\phi_{k}\left|\frac{1}{r_{i j}} \mathbf{r}^{T}\left(\underline{\mathbf{E}}_{i j}-\underline{\mathbf{E}}_{j j}\right) \underline{\tilde{\mathbf{A}}}_{l}\left(\mathbf{r}-\tilde{\mathbf{s}}_{l}\right)\right| \tilde{\phi}_{l}\right\rangle+4\left\langle\phi_{k}\left|\frac{1}{r_{i j}} \mathbf{r}^{T}\left(\underline{\mathbf{E}}_{i j}-\underline{\mathbf{E}}_{j j}\right) \underline{\tilde{\mathbf{A}}}_{l} \underline{\mathbf{E}}_{j i} \underline{\tilde{\mathbf{A}}}_{l}\left(\mathbf{r}-\tilde{\mathbf{s}}_{l}\right)\right| \tilde{\phi}_{l}\right\rangle+ \\
+4\left\langle\phi_{k}\left|\frac{1}{r_{i j}}\left(\mathbf{r}-\tilde{\mathbf{s}}_{l}\right)^{T} \underline{\tilde{\mathbf{A}}}_{l} \underline{\mathbf{E}}_{j i} \underline{\tilde{\mathbf{A}}}_{l}\left(\underline{\mathbf{E}}_{i j}-\underline{\mathbf{E}}_{j j}\right)^{T} \mathbf{r}\right| \tilde{\phi}_{l}\right\rangle-8\left\langle\phi_{k}\left|\frac{1}{r_{i j}}\left(\mathbf{r}-\tilde{\mathbf{s}}_{l}\right)^{T} \underline{\tilde{\mathbf{A}}}_{l} \underline{\mathbf{E}}_{j i} \underline{\tilde{\mathbf{A}}}_{l}\left(\mathbf{r}-\tilde{\mathbf{s}}_{l}\right) \mathbf{r}^{T}\left(\underline{\mathbf{E}}_{i j}-\underline{\mathbf{E}}_{j j}\right) \underline{\tilde{\mathbf{s}}}_{l}\left(\mathbf{r}-\tilde{\mathbf{s}}_{l}\right)\right| \tilde{\phi}_{l}\right\rangle .
\end{gathered}
$$

\section{INTEGRALS}

Now we calculate the integrals appearing in term 1, term 2 , and term 3 . The integrals are as follows:

1. $\left\langle\phi_{k}\left|\frac{1}{r_{i j}}\left(\mathbf{r}^{T} \underline{\mathbf{X}} \mathbf{r}\right)\left(\mathbf{r}^{T} \underline{\mathbf{Y}} \mathbf{r}\right)\right| \tilde{\phi}_{l}\right\rangle$,

2. $\left\langle\phi_{k}\left|\frac{1}{r_{i j}}\left(\underline{\mathbf{X} \mathbf{s}_{n}}\right)^{T} \mathbf{r}\left(\underline{\mathbf{Y}} \mathbf{s}_{m}\right)^{T} \mathbf{r}\right| \tilde{\phi}_{l}\right\rangle$, where $m, n$ denotes $k$ or $l$,

3. $\left\langle\phi_{k}\left|\frac{1}{r_{i j}}\left(\mathbf{r}^{T} \underline{\mathbf{X}} \mathbf{r}\right)\left(\underline{\mathbf{Y}} \mathbf{s}_{m}\right)^{T} \mathbf{r}\right| \tilde{\phi}_{l}\right\rangle$, where $m$ denotes $k$ or $l$.

The integrals are determined next.

A. Integral $\left\langle\phi_{k}\left|\frac{1}{r_{i j}}\left(\mathbf{r}^{T} \underline{\mathbf{X}} \mathbf{r}\right)\left(\mathbf{r}^{T} \underline{\mathbf{Y}} \mathbf{r}\right)\right| \tilde{\phi}_{I}\right\rangle$

$$
\begin{aligned}
\left\langle\phi_{k}\left|\frac{1}{r_{i j}}\left(\mathbf{r}^{T} \underline{\mathbf{X}}\right)\left(\mathbf{r}^{T} \underline{\mathbf{Y}}\right)\right| \tilde{\phi}_{l}\right\rangle= & \frac{2}{\sqrt{\pi}} \exp \left[\gamma-\mathbf{s}^{T} \underline{\mathbf{A}}_{k l} \mathbf{s}\right] \partial_{\alpha} \partial_{\beta} \int_{0}^{\infty} d t \int d \mathbf{r} \\
& \times\left.\exp \left[-\mathbf{r}^{T}\left(\underline{\mathbf{A}}_{k l}+t^{2} \underline{\mathbf{J}}_{i j}+\alpha \underline{\mathbf{X}}+\beta \underline{\mathbf{Y}}\right) \mathbf{r}+2 \mathbf{s}^{T} \underline{\mathbf{A}}_{k l} \mathbf{r}\right]\right|_{\alpha=\beta=0}
\end{aligned}
$$


224111-7 Stank et al.

J. Chem. Phys. 145, 224111 (2016)

$$
\begin{aligned}
= & \frac{2}{\sqrt{\pi}} \exp \left[\gamma-\mathbf{s}^{T} \underline{\mathbf{A}}_{k l} \mathbf{s}\right] \pi^{3 n / 2}\left|\mathbf{A}_{k l}\right|^{-3 / 2} \\
& \times\left.\partial_{\alpha} \partial_{\beta} \int_{0}^{\infty} d t\left|\underline{\mathbf{I}}+t^{2} \underline{\mathbf{J}}_{i j} \underline{\mathbf{A}}_{k l}^{-1}+\alpha \underline{\mathbf{X}}_{k l}^{-1}+\beta \underline{\mathbf{Y A}}_{k l}^{-1}\right|^{-1 / 2} \exp \left[\mathbf{s}^{T} \underline{\mathbf{A}}_{k l}\left(\underline{\mathbf{A}}_{k l}+t^{2} \underline{\mathbf{J}}_{i j}+\alpha \underline{\mathbf{X}}+\beta \underline{\mathbf{Y}}\right)^{-1} \underline{\mathbf{A}}_{k l} \mathbf{\mathbf { s }}\right]\right|_{\alpha=\beta=0}
\end{aligned}
$$

The integrant in (40) is calculated using the following transformations:

1. First we introduce the following quantities:

- $\left|\mathbf{I}+t^{2} \mathbf{J}_{i j} \mathbf{A}_{k l}^{-1}+\alpha \mathbf{X} \mathbf{A}_{k l}^{-1}+\beta \mathbf{Y} \mathbf{A}_{k l}^{-1}\right| \equiv|\mathbf{W}|$,

- $\left(\underline{\mathbf{A}}_{k l}+t^{2} \underline{\mathbf{J}}_{i j}+\alpha \underline{\mathbf{X}}+\beta \underline{\mathbf{Y}}\right)^{-1} \equiv \underline{\mathbf{M}}^{-1}$.

2. Using (23), (24), and (26) we can rewrite expression (40) as

$$
\begin{aligned}
& \left.\partial_{\alpha} \partial_{\beta}\left\{\left|\mathbf{I}+t^{2} \mathbf{J}_{i j} \mathbf{A}_{k l}^{-1}+\alpha \mathbf{X} \mathbf{A}_{k l}^{-1}+\beta \mathbf{Y} \mathbf{A}_{k l}^{-1}\right|^{-3 / 2} \exp \left[\mathbf{s}^{T} \underline{\mathbf{A}}_{k l}^{-1}\left(\underline{\mathbf{A}}_{k l}+t^{2} \underline{\mathbf{J}}_{i j}+\alpha \underline{\mathbf{X}}+\beta \underline{\mathbf{Y}}\right)^{-1} \underline{\mathbf{A}}_{k l}^{-1} \mathbf{s}\right]\right\}\right|_{\alpha=\beta=0} \\
& \quad=\left.\partial_{\alpha} \partial_{\beta}\left\{|\mathbf{W}|^{-3 / 2} \exp \left[\mathbf{e}^{T} \underline{\mathbf{M}}^{-1} \mathbf{e}\right]\right\}\right|_{\alpha=\beta=0} \\
& \quad=\left|\mathbf{I}+t^{2} \mathbf{J}_{i j} \mathbf{A}_{k l}^{-1}\right|^{-3 / 2} \exp \left[\mathbf{e}^{T}\left(\underline{\mathbf{A}}_{k l}+t^{2} \underline{\mathbf{J}}_{i j}\right)^{-1} \mathbf{e}\right]\left\{w_{0}-\frac{t^{2}}{1+t^{2} a} w_{2}+\frac{t^{4}}{\left(1+t^{2} a\right)^{2}} w_{4}-\frac{t^{6}}{\left(1+t^{2} a\right)^{3}} w_{6}+\frac{t^{8}}{\left(1+t^{2} a\right)^{4}} w_{8}\right\}
\end{aligned}
$$

where

- $a \equiv \operatorname{Tr}\left[\mathbf{J}_{i j} \mathbf{A}_{k l}^{-1}\right]$,

- $w_{0}=\frac{9}{4} a_{1} b_{1}+\frac{3}{2}\left(c_{1}+b_{1} d_{1}+a_{1} e_{1}\right)+f_{1}+d_{1} e_{1}$,

- $w_{2}=\frac{9}{4}\left(a_{1} b_{2}+a_{2} b_{1}\right)+\frac{3}{2}\left(c_{2}+b_{1} d_{2}+b_{2} d_{1}+a_{1} e_{2}+a_{2} e_{1}\right)+f_{2}+d_{1} e_{2}+d_{2} e_{1}$,

- $w_{4}=\frac{9}{4} a_{2} b_{2}+\frac{3}{2}\left(c_{3}+b_{1} d_{3}+b_{2} d_{2}+a_{1} e_{3}+a_{2} e_{2}\right)+f_{3}+d_{1} e_{3}+d_{3} e_{1}+d_{2} e_{2}$,

- $w_{6}=\frac{3}{2}\left(b_{2} d_{3}+a_{2} e_{3}\right)+f_{4}+d_{2} e_{3}+d_{3} e_{2}$,

- $w_{8}=d_{3} e_{3}$,

and

$$
\begin{aligned}
& a_{1}=\operatorname{Tr}\left[\mathbf{X} \mathbf{A}_{k l}^{-1}\right] \text {, } \\
& a_{2}=\operatorname{Tr}\left[\mathbf{J}_{i j} \mathbf{A}_{k l}^{-1} \mathbf{X} \mathbf{A}_{k l}^{-1}\right] \text {, } \\
& b_{1}=\operatorname{Tr}\left[\mathbf{Y} \mathbf{A}_{k l}^{-1}\right] \text {, } \\
& b_{2}=\operatorname{Tr}\left[\mathbf{J}_{i j} \mathbf{A}_{k l}^{-1} \mathbf{Y} \mathbf{A}_{k l}^{-1}\right] \text {, } \\
& c_{1}=\operatorname{Tr}\left[\mathbf{X} \mathbf{A}_{k l}^{-1} \mathbf{Y} \mathbf{A}_{k l}^{-1}\right] \text {, } \\
& c_{2}=\operatorname{Tr}\left[\mathbf{J}_{i j} \mathbf{A}_{k l}^{-1} \mathbf{X} \mathbf{A}_{k l}^{-1} \mathbf{Y} \mathbf{A}_{k l}^{-1}\right]+\operatorname{Tr}\left[\mathbf{X} \mathbf{A}_{k l}^{-1} \mathbf{J}_{i j} \mathbf{A}_{k l}^{-1} \mathbf{Y} \mathbf{A}_{k l}^{-1}\right], \\
& c_{3}=\operatorname{Tr}\left[\mathbf{J}_{i j} \mathbf{A}_{k l}^{-1} \mathbf{X} \mathbf{A}_{k l}^{-1} \mathbf{J}_{i j} \mathbf{A}_{k l}^{-1} \mathbf{Y} \mathbf{A}_{k l}^{-1}\right] \text {, } \\
& d_{1}=\mathbf{s}^{T} \underline{\mathbf{X}} \mathbf{s}, \\
& d_{2}=\left(\mathbf{s}^{T} \underline{\mathbf{J}}_{i j} \underline{\mathbf{A}}_{k l}^{-1} \underline{\mathbf{X}} \mathbf{s}\right)+\left(\mathbf{s}^{T} \underline{\mathbf{X}}_{k l}^{-1} \underline{\mathbf{J}}_{i j} \mathbf{s}\right)=2\left(\mathbf{s}^{T} \underline{\mathbf{J}}_{i j} \underline{\mathbf{A}}_{k l}^{-1} \underline{\mathbf{X}} \mathbf{s}\right), \\
& d_{3}=\left(\mathbf{s}^{T} \underline{\mathbf{J}}_{i j} \underline{\mathbf{A}}_{k l}^{-1} \underline{\mathbf{X}}_{k l}^{-1} \underline{\mathbf{J}}_{i j} \mathbf{s}\right), \\
& e_{1}=\mathbf{s}^{T} \underline{\mathbf{Y}} \mathbf{s}, \\
& e_{2}=\left(\mathbf{s}^{T} \underline{\mathbf{J}}_{i j} \underline{\mathbf{A}}_{k l}^{-1} \underline{\mathbf{Y}} \mathbf{s}\right)+\left(\mathbf{s}^{T} \underline{\mathbf{Y}} \underline{\mathbf{A}}_{k l}^{-1} \underline{\mathbf{J}}_{i j} \mathbf{s}\right)=2\left(\mathbf{s}^{T} \underline{\mathbf{J}}_{i j} \underline{\mathbf{A}}_{k l}^{-1} \underline{\mathbf{Y}} \mathbf{s}\right), \\
& e_{3}=\left(\mathbf{s}^{T} \underline{\mathbf{J}}_{i j} \underline{\mathbf{A}}_{k l}^{-1} \underline{\mathbf{Y A}}_{k l}^{-1} \underline{\mathbf{J}}_{i j} \mathbf{s}\right) \text {, } \\
& f_{1}=\mathbf{s}^{T} \underline{\mathbf{X}}_{k l}^{-1} \underline{\mathbf{Y}} \mathbf{s}+\mathbf{s}^{T} \underline{\mathbf{Y A}}{ }_{k l}^{-1} \underline{\mathbf{X}} \mathbf{s}=2 \mathbf{s}^{T} \underline{\mathbf{X}} \underline{\mathbf{A}}_{k l}^{-1} \underline{\mathbf{Y}} \mathbf{s}, \\
& f_{2}=\left(\mathbf{s}^{T} \underline{\mathbf{X A}}_{k l}^{-1} \underline{\mathbf{J}}_{i j} \underline{\mathbf{A}}_{k l}^{-1} \underline{\mathbf{Y}} \mathbf{s}\right)+\left(\mathbf{s}^{T} \underline{\mathbf{J}}_{i j} \underline{\mathbf{A}}_{k l}^{-1} \underline{\mathbf{X A}}_{k l}^{-1} \underline{\mathbf{Y}} \mathbf{\mathbf { s }}\right)+\left(\mathbf{s}^{T} \underline{\mathbf{X A}}_{k l}^{-1} \underline{\mathbf{Y A}}_{k l}^{-1} \underline{\mathbf{J}}_{i j} \mathbf{s}\right) \\
& +\left(\mathbf{s}^{T} \underline{\mathbf{Y A}}_{k l}^{-1} \underline{\mathbf{J}}_{i j} \underline{\mathbf{A}}_{k l}^{-1} \underline{\mathbf{X}} \mathbf{s}\right)+\left(\mathbf{s}^{T} \underline{\mathbf{J}}_{i j} \underline{\mathbf{A}}_{k l}^{-1} \underline{\mathbf{Y A}}_{k l}^{-1} \underline{\mathbf{X}} \mathbf{s}\right)+\left(\mathbf{s}^{T} \underline{\mathbf{Y A}}_{k l}^{-1} \underline{\mathbf{X A}}_{k l}^{-1} \underline{\mathbf{J}}_{i j} \mathbf{s}\right),
\end{aligned}
$$




$$
\begin{aligned}
f_{3}= & \left(\mathbf{s}^{T} \underline{\mathbf{X}}_{k l}^{-1} \underline{\mathbf{J}}_{i j} \underline{\mathbf{A}}_{k l}^{-1} \underline{\mathbf{Y}}_{k l}^{-1} \underline{\mathbf{J}}_{i j} \mathbf{s}\right)+\left(\mathbf{s}^{T} \underline{\mathbf{J}}_{i j} \underline{\mathbf{A}}_{k l}^{-1} \underline{\mathbf{X}}_{k l}^{-1} \underline{\mathbf{J}}_{i j} \underline{\mathbf{A}}_{k l}^{-1} \underline{\mathbf{Y}} \mathbf{s}\right) \\
& +\left(\mathbf{s}^{T} \underline{\mathbf{J}}_{i j} \underline{\mathbf{A}}_{k l}^{-1} \underline{\mathbf{X}}_{k l}^{-1} \underline{\mathbf{Y}}_{k l}^{-1} \underline{\mathbf{J}}_{i j} \mathbf{s}\right)+\left(\mathbf{s}^{T} \underline{\mathbf{Y}}_{k l}^{-1} \underline{\mathbf{J}}_{i j} \underline{\mathbf{A}}_{k l}^{-1} \underline{\mathbf{X}}_{k l}^{-1} \underline{\mathbf{J}}_{i j} \mathbf{s}\right) \\
& +\left(\mathbf{s}^{T} \underline{\mathbf{J}}_{i j} \underline{\mathbf{A}}_{k l}^{-1} \underline{\mathbf{Y A}}_{k l}^{-1} \underline{\mathbf{J}}_{i j} \underline{\mathbf{A}}_{k l}^{-1} \underline{\mathbf{X}}^{\mathbf{s}}\right)+\left(\mathbf{s}^{T} \underline{\mathbf{J}}_{i j} \underline{\mathbf{A}}_{k l}^{-1} \underline{\mathbf{Y A}}_{k l}^{-1} \underline{\mathbf{X}}_{k l}^{-1} \underline{\mathbf{J}}_{i j} \mathbf{s}\right), \\
f_{4}= & \left(\mathbf{s}^{T} \underline{\mathbf{J}}_{i j} \underline{\mathbf{A}}_{k l}^{-1} \underline{\mathbf{X}}_{k l}^{-1} \underline{\mathbf{J}}_{i j} \underline{\mathbf{A}}_{k l}^{-1} \underline{\mathbf{Y}}_{k l}^{-1} \underline{\mathbf{J}}_{i j} \mathbf{s}\right)+\left(\mathbf{s}^{T} \underline{\mathbf{J}}_{i j} \underline{\mathbf{A}}_{k l}^{-1} \underline{\mathbf{Y A}}_{k l}^{-1} \underline{\mathbf{J}}_{i j} \underline{\mathbf{A}}_{k l}^{-1} \underline{\mathbf{X}}_{k l}^{-1} \mathbf{J}_{i j} \mathbf{s}\right) .
\end{aligned}
$$

Hence, for $\beta=\mathbf{s}^{T} \underline{\mathbf{J}}_{i j} \mathbf{s}$ the integral (40) simplifies to

$$
\begin{aligned}
\left\langle\phi_{k}\left|\frac{1}{r_{i j}}\left(\mathbf{r}^{T} \underline{\mathbf{X r}}\right)\left(\mathbf{r}^{T} \underline{\mathbf{Y}} \mathbf{r}\right)\right| \tilde{\phi}_{l}\right\rangle= & \frac{2}{\sqrt{\pi}} \pi^{3 n / 2} \exp [\gamma]\left|\mathbf{A}_{k l}\right|^{-3 / 2} \int_{0}^{\infty} d t \exp \left[-\frac{t^{2} \beta}{1+t^{2} a}\right] \\
& \times\left\{\frac{1}{\left(1+t^{2} a\right)^{3 / 2}} w_{0}-\frac{t^{2}}{\left(1+t^{2} a\right)^{5 / 2}} w_{2}+\frac{t^{4}}{\left(1+t^{2} a\right)^{7 / 2}} w_{4}-\frac{t^{6}}{\left(1+t^{2} a\right)^{9 / 2}} w_{6}+\frac{t^{8}}{\left(1+t^{2} a\right)^{11 / 2}} w_{8}\right\}= \\
& \equiv \frac{2}{\sqrt{\pi}} \pi^{3 n / 2} \exp [\gamma]\left|\mathbf{A}_{k l}\right|^{-3 / 2}\left(I_{0}-I_{2}+I_{4}-I_{6}+I_{8}\right) .
\end{aligned}
$$

One-dimensional integrals $I_{i}(i=0,2,4,6$, and 8$)$ are calculated as follows:

$$
\begin{gathered}
I_{0}=\int_{0}^{\infty} d t \exp \left[-\frac{t^{2}}{1+t^{2} a} \beta\right] \frac{1}{\left(1+t^{2} a\right)^{3 / 2}} w_{0}=\frac{1}{2} \sqrt{\frac{\pi}{\beta}} \operatorname{Erf}\left[\sqrt{\frac{\alpha}{\beta}}\right] w_{0}=\frac{1}{\sqrt{a}} \mathrm{~F}_{0}\left(\frac{\beta}{a}\right) w_{0}, \\
I_{2}=\int_{0}^{\infty} d t \exp \left[-\frac{t^{2}}{1+t^{2} a} \beta\right] \frac{t^{2}}{\left(1+t^{2} a\right)^{5 / 2}} w_{2}=-\frac{1}{a^{3 / 2}} \mathrm{~F}_{1}\left(\frac{\beta}{a}\right) w_{2}, \\
I_{4}=\int_{0}^{\infty} d t \exp \left[-\frac{t^{2}}{1+t^{2} a} \beta\right] \frac{t^{4}}{\left(1+t^{2} a\right)^{7 / 2}} w_{4}=\frac{1}{a^{5 / 2}} \mathrm{~F}_{2}\left(\frac{\beta}{a}\right) w_{4}, \\
I_{6}=\int_{0}^{\infty} d t \exp \left[-\frac{t^{2}}{1+t^{2} a} \beta\right] \frac{t^{6}}{\left(1+t^{2} a\right)^{9 / 2}} w_{6}=-\frac{1}{a^{7 / 2}} \mathrm{~F}_{3}\left(\frac{\beta}{a}\right) w_{6}, \\
I_{8}=\int_{0}^{\infty} d t \exp \left[-\frac{t^{2}}{1+t^{2} a} \beta\right] \frac{t^{8}}{\left(1+t^{2} a\right)^{11 / 2}} w_{8}=\frac{1}{a^{9 / 2}} \mathrm{~F}_{4}\left(\frac{\beta}{a}\right) w_{8},
\end{gathered}
$$

where

$$
\begin{aligned}
& \mathrm{F}_{1}\left(\frac{\beta}{a}\right)=\frac{1}{2} \frac{a}{\beta}\left(\exp \left[-\frac{\beta}{a}\right]-\mathrm{F}_{0}\left(\frac{\beta}{a}\right)\right), \\
& \mathrm{F}_{2}\left(\frac{\beta}{a}\right)=-\frac{3}{4} \frac{a^{2}}{\beta^{2}}\left(\exp \left[-\frac{\beta}{a}\right]+\frac{2}{3} \frac{\beta}{a} \exp \left[-\frac{\beta}{a}\right]-\mathrm{F}_{0}\left(\frac{\beta}{a}\right)\right), \\
& \mathrm{F}_{3}\left(\frac{\beta}{a}\right)=\frac{15}{8} \frac{a^{3}}{\beta^{3}}\left(\exp \left[-\frac{\beta}{a}\right]+\frac{2}{3} \frac{\beta}{a} \exp \left[-\frac{\beta}{a}\right]+\frac{4}{15} \frac{\beta^{2}}{a^{2}} \exp \left[-\frac{\beta}{a}\right]-\mathrm{F}_{0}\left(\frac{\beta}{a}\right)\right), \\
& \mathrm{F}_{4}\left(\frac{\beta}{a}\right)=-\frac{105}{16} \frac{a^{4}}{\beta^{4}}\left(\exp \left[-\frac{\beta}{a}\right]+\frac{2}{3} \frac{\beta}{a} \exp \left[-\frac{\beta}{a}\right]+\frac{4}{15} \frac{\beta^{2}}{a^{2}} \exp \left[-\frac{\beta}{a}\right]+\frac{8}{105} \frac{\beta^{3}}{a^{3}} \exp \left[-\frac{\beta}{a}\right]-\mathrm{F}_{0}\left(\frac{\beta}{a}\right)\right) .
\end{aligned}
$$

So finally

$$
\left\langle\phi_{k}\left|\frac{1}{r_{i j}}\left(\mathbf{r}^{T} \underline{\mathbf{X}} \mathbf{r}\right)\left(\mathbf{r}^{T} \underline{\mathbf{Y}}\right)\right| \tilde{\phi}_{l}\right\rangle=\frac{2}{\sqrt{\pi a}}\left\langle\phi_{k} \mid \tilde{\phi}_{l}\right\rangle\left\{\mathrm{F}_{0}\left(\frac{\beta}{a}\right) w_{0}+\frac{1}{a} \mathrm{~F}_{1}\left(\frac{\beta}{a}\right) w_{2}+\frac{1}{a^{2}} \mathrm{~F}_{2}\left(\frac{\beta}{a}\right) w_{4}+\frac{1}{a^{3}} \mathrm{~F}_{3}\left(\frac{\beta}{a}\right) w_{6}+\frac{1}{a^{4}} \mathrm{~F}_{4}\left(\frac{\beta}{a}\right) w_{8}\right\},
$$

where $\left\langle\phi_{k} \mid \tilde{\phi}_{l}\right\rangle$ is the overlap integral. 


\section{B. Integral $\left\langle\phi_{k}\left|\frac{1}{r_{i j}}\left(\underline{X} s_{n}\right)^{T} \mathbf{r}\left(\underline{Y} s_{m}\right)^{T} \mathbf{r}\right| \tilde{\phi}_{I}\right\rangle$}

$$
\begin{aligned}
\left\langle\phi_{k}\left|\frac{1}{r_{i j}}\left(\underline{\mathbf{X}}_{n}\right)^{T} \mathbf{r}\left(\underline{\mathbf{Y}}_{m}\right)^{T} \mathbf{r}\right| \tilde{\phi}_{l}\right\rangle= & \frac{2}{\sqrt{\pi}} \exp \left[\gamma-\mathbf{s}^{T} \underline{\mathbf{A}}_{k l} \mathbf{s}\right] \partial_{\alpha} \partial_{\beta} \int_{0}^{\infty} d t \int d \mathbf{r} \\
& \times\left.\exp \left[-\mathbf{r}^{T}\left(\underline{\mathbf{A}}_{k l}+t^{2} \underline{\mathbf{J}}_{i j}\right) \mathbf{r}+2\left(\underline{\mathbf{A}}_{k l} \mathbf{s}+\frac{\alpha}{2} \underline{\mathbf{X}}_{n}+\frac{\beta}{2} \underline{\mathbf{Y}}_{m}\right)^{T} \mathbf{r}\right]\right|_{\alpha=\beta=0} \\
= & \left.\frac{2}{\sqrt{\pi}}\left\langle\phi_{k} \mid \tilde{\phi}_{l}\right\rangle \exp \left[-\mathbf{s}^{T} \underline{\mathbf{A}}_{k l} \mathbf{s}\right] \partial_{\alpha} \partial_{\beta} \int_{0}^{\infty} d t\left(1+t^{2} a\right)^{-3 / 2} \exp \left[\mathbf{m}^{T} \underline{\mathbf{M}}^{-1} \mathbf{m}\right]\right|_{\alpha=\beta=0},
\end{aligned}
$$

where $\mathbf{m} \equiv\left(\underline{\mathbf{A}}_{k l} \mathbf{s}+\frac{\alpha}{2} \underline{\mathbf{F}} \mathbf{s}_{n}+\frac{\beta}{2} \underline{\mathbf{C}} \mathbf{s}_{m}\right)$ and $\underline{\mathbf{M}}=\underline{\mathbf{A}}_{k l}+t^{2} \underline{\mathbf{J}}_{i j}$. Using (24) and (26) to transform the integrand expression in (51), the integral is equal to

$$
\left\langle\phi_{k}\left|\frac{1}{r_{i j}}\left(\underline{\mathbf{X}}_{n}\right)^{T}\left(\underline{\mathbf{Y}} \mathbf{s}_{m}\right)^{T}\right| \tilde{\phi}_{l}\right\rangle=\frac{1}{4} \frac{2}{\sqrt{\pi}}\left\langle\phi_{k} \mid \phi_{l}\right\rangle \frac{1}{\sqrt{a}}\left(\mathrm{~F}_{0}\left(\frac{\beta}{a}\right) w_{0}+\frac{1}{a} \mathrm{~F}_{1}\left(\frac{\beta}{a}\right) w_{1}+\frac{1}{a^{2}} \mathrm{~F}_{2}\left(\frac{\beta}{a}\right) w_{2}\right),
$$

where

- $\beta=\mathbf{s}^{T} \mathbf{J}_{i j} \mathbf{s}$,

- $w_{0}=\left(\mathbf{s}_{n}^{T} \underline{\mathbf{F}}^{T} \underline{\mathbf{A}}_{k l}^{-1} \underline{\mathbf{C}}_{m}\right)+\left(\mathbf{s}_{m}^{T} \underline{\mathbf{C}}^{T} \underline{\mathbf{A}}_{k l}^{-1} \underline{\mathbf{F}}_{n}\right)+\left(\left(\mathbf{s}^{T} \underline{\mathbf{C}}_{m}\right)+\left(\mathbf{s}_{m}^{T} \underline{\mathbf{C}}^{T} \mathbf{s}\right)\right)\left(\left(\mathbf{s}^{T} \underline{\mathbf{F}}_{n}\right)+\left(\mathbf{s}_{n}^{T} \underline{\mathbf{F}}^{T} \mathbf{s}\right)\right)$,

- $w_{1}=\left(\mathbf{s}_{n}^{T} \underline{\mathbf{F}}^{T} \underline{\mathbf{A}}_{k l}^{-1} \underline{\mathbf{J}}_{i j} \underline{\mathbf{A}}_{k l}^{-1} \underline{\mathbf{C}}_{m}\right)+\left(\mathbf{s}_{m}^{T} \underline{\mathbf{C}}^{T} \underline{\mathbf{A}}_{k l}^{-1} \underline{\mathbf{J}}_{i j} \underline{\mathbf{A}}_{k l}^{-1} \underline{\mathbf{F}}_{n}\right)+\left(\left(\mathbf{s}^{T} \underline{\mathbf{C}}_{m}\right)+\left(\mathbf{s}_{m}^{T} \underline{\mathbf{C}}^{T} \mathbf{s}\right)\right)\left(\left(\mathbf{s}^{T} \underline{\mathbf{J}}_{i j} \underline{\mathbf{A}}_{k l}^{-1} \underline{\mathbf{F}}_{m}\right)+\left(\mathbf{s}_{m}^{T} \underline{\mathbf{F}}^{T} \underline{\mathbf{A}}_{k l}^{-1} \underline{\mathbf{J}}_{i j} \mathbf{s}\right)\right)$ $+\left(\left(\mathbf{s}^{T} \underline{\mathbf{F}}_{m}\right)+\left(\mathbf{s}_{m}^{T} \underline{\mathbf{F}}^{T} \mathbf{s}\right)\right)\left(\left(\mathbf{s}^{T} \underline{\mathbf{J}}_{i j} \underline{\mathbf{A}}_{k l}^{-1} \underline{\mathbf{C}}_{m}\right)+\left(\mathbf{s}_{m}^{T} \underline{\mathbf{C}}^{T} \underline{\mathbf{A}}_{k l}^{-1} \underline{\mathbf{J}}_{i j} \mathbf{s}\right)\right)$,

- $w_{2}=\left(\left(\mathbf{s}^{T} \underline{\mathbf{J}}_{i j} \underline{\mathbf{A}}_{k l}^{-1} \underline{\mathbf{C}}_{m}\right)+\left(\mathbf{s}_{m}^{T} \underline{\mathbf{C}}^{T} \underline{\mathbf{A}}_{k l}^{-1} \underline{\mathbf{J}}_{i j} \mathbf{s}\right)\right) \cdot\left(\left(\mathbf{s}^{T} \underline{\mathbf{J}}_{i j} \underline{\mathbf{A}}_{k l}^{-1} \underline{\mathbf{F}}_{m}\right)+\left(\mathbf{s}_{m}^{T} \underline{\mathbf{F}}^{T} \underline{\mathbf{A}}_{k l}^{-1} \underline{\mathbf{J}}_{i j} \mathbf{s}\right)\right)$.

\section{Integral $\left\langle\phi_{k}\left|\frac{1}{r_{i j}}\left(\mathbf{r}^{T} \underline{\mathbf{X r}}\right)\left(\underline{\mathbf{Y}} \mathbf{s}_{m}\right)^{T} \mathbf{r}\right| \tilde{\phi}_{l}\right\rangle$}

$$
\begin{aligned}
\left\langle\phi_{k}\left|\frac{1}{r_{i j}}\left(\mathbf{r}^{T} \underline{\mathbf{X}} \mathbf{r}\right)\left(\underline{\mathbf{Y}} \mathbf{s}_{m}\right)^{T} \mathbf{r}\right| \tilde{\phi}_{l}\right\rangle= & -\frac{2}{\sqrt{\pi}} \exp \left[\gamma-\mathbf{s}^{T} \underline{\mathbf{A}}_{k l} \mathbf{s}\right] \partial_{\alpha} \partial_{\beta} \int_{0}^{\infty} d t \int d \mathbf{r} \\
& \times\left.\exp \left[-\mathbf{r}^{T}\left(\underline{\mathbf{A}}_{k l}+t^{2} \underline{\mathbf{J}}_{i j}+\alpha \underline{\mathbf{X}}\right) \mathbf{r}+2\left(\underline{\mathbf{A}}_{k l} \mathbf{s}+\frac{\beta}{2} \underline{\mathbf{Y}}_{m}\right)^{T} \mathbf{r}\right]\right|_{\alpha=\beta=0} \\
= & \frac{1}{\sqrt{\pi}}\left\langle\phi_{k} \mid \tilde{\phi}_{l}\right\rangle \frac{1}{\sqrt{a}}\left\{\mathrm{~F}_{0}\left(\frac{\beta}{a}\right) w_{0}+\frac{1}{a} \mathrm{~F}_{1}\left(\frac{\beta}{a}\right) w_{2}+\frac{1}{a^{2}} \mathrm{~F}_{2}\left(\frac{\beta}{a}\right) w_{4}+\frac{1}{a^{3}} \mathrm{~F}_{3}\left(\frac{\beta}{a}\right) w_{6}\right\},
\end{aligned}
$$

where the quantities appearing in the above equation have the following meaning:

$$
\begin{aligned}
& a=\operatorname{Tr}\left[\mathbf{J}_{i j} \mathbf{A}_{k l}^{-1}\right], \quad b=\operatorname{Tr}\left[\mathbf{J}_{i j} \mathbf{A}_{k l}^{-1} \mathbf{X} \mathbf{A}_{k l}^{-1}\right], \quad \tilde{a}=\operatorname{Tr}\left[\mathbf{A}_{k l}^{-1} \mathbf{X}\right], \\
& w_{0}=\frac{3}{2} \tilde{a}\left(\mathbf{s}^{T} \underline{\mathbf{Y}} \mathbf{s}_{m}\right)+\left(\mathbf{s}^{T} \underline{\mathbf{X A}}_{k l}^{-1} \underline{\mathbf{Y}} \mathbf{s}_{m}\right)+\left(\mathbf{s}^{T} \underline{\mathbf{Y}} \mathbf{s}_{m}\right)\left(\mathbf{s}^{T} \underline{\mathbf{X}} \mathbf{s}\right) \\
& w_{2}=\frac{3}{2} \tilde{a}\left(\mathbf{s}^{T} \underline{\mathbf{J}}_{i j} \underline{\mathbf{A}}_{k l}^{-1} \underline{\mathbf{Y}}_{m}\right)+\frac{3}{2} b\left(\mathbf{s}^{T} \underline{\mathbf{Y}} \mathbf{s}_{m}\right)+\left(\mathbf{s}^{T} \underline{\mathbf{X A}}_{k l}^{-1} \underline{\mathbf{J}}_{i j} \underline{\mathbf{A}}_{k l}^{-1} \underline{\mathbf{Y}}_{m}\right)+\left(\mathbf{s}^{T} \underline{\mathbf{J}}_{i j} \underline{\mathbf{A}}_{k l}^{-1} \underline{\mathbf{X A}}_{k l}^{-1} \underline{\mathbf{Y}}_{m}\right) \\
& +2\left(\mathbf{s}^{T} \underline{\mathbf{Y}} \mathbf{s}_{m}\right)\left(\mathbf{s}^{T} \underline{\mathbf{J}}_{i j} \underline{\mathbf{A}}_{k l}^{-1} \underline{\mathbf{X}} \mathbf{s}\right)+\left(\mathbf{s}^{T} \underline{\mathbf{X}} \mathbf{s}\right)\left(\mathbf{s}^{T} \underline{\mathbf{J}}_{i j} \underline{\mathbf{A}}_{k l}^{-1} \underline{\mathbf{Y}} \mathbf{s}_{m}\right) \\
& w_{4}=\frac{3}{2} b\left(\mathbf{s}^{T} \underline{\mathbf{J}}_{i j} \underline{\mathbf{A}}_{k l}^{-1} \underline{\mathbf{Y}}_{m}\right)+\left(\mathbf{s}^{T} \underline{\mathbf{J}}_{i j} \underline{\mathbf{A}}_{k l}^{-1} \underline{\mathbf{X}} \underline{\mathbf{A}}_{k l}^{-1} \underline{\mathbf{J}}_{i j} \underline{\mathbf{A}}_{k l}^{-1} \underline{\mathbf{Y}} \mathbf{s}_{m}\right) \\
& +\left(\mathbf{s}^{T} \underline{\mathbf{Y}}_{m}\right)\left(\mathbf{s}^{T} \underline{\mathbf{J}}_{i j} \underline{\mathbf{A}}_{k l}^{-1} \underline{\mathbf{X}}_{k l}^{-1} \underline{\mathbf{J}}_{i j} \mathbf{s}\right)+2\left(\mathbf{s}^{T} \underline{\mathbf{J}}_{i j} \underline{\mathbf{A}}_{k l}^{-1} \underline{\mathbf{Y}}_{m}\right)\left(\mathbf{s}^{T} \underline{\mathbf{J}}_{i j} \underline{\mathbf{A}}_{k l}^{-1} \underline{\mathbf{X}} \mathbf{s}\right) \\
& w_{6}=\left(\mathbf{s}^{T} \underline{\mathbf{J}}_{i j} \underline{\mathbf{A}}_{k l}^{-1} \underline{\mathbf{Y}} \mathbf{s}_{m}\right)\left(\mathbf{s}^{T} \underline{\mathbf{J}}_{i j} \underline{\mathbf{A}}_{k l}^{-1} \underline{\mathbf{X A}}_{k l}^{-1} \underline{\mathbf{J}}_{i j} \mathbf{s}\right) \text {. }
\end{aligned}
$$

\section{NUMERICAL TESTS}

The formulas derived in this work are implemented in a computer code written in FORTRAN 90 and employing the Message Passing Interface (MPI) protocol. In the first step, the code is tested in the calculations for the $\mathrm{H}_{2}$ molecule performed at 12 selected internuclear distances ranging from 0.4 to 19.5 a.u. with 1000 ECGs. At each distance, the non-linear parameters of the Gaussians (i.e., the $\mathbf{L}_{k}$ matrix elements and the elements of the $\mathbf{s}_{k}$ vector of the Gaussian centers) are 
TABLE I. Non-relativistic electronic ground-state energies and orbit-orbit relativistic corrections $\left(\left\langle\hat{H}_{\mathrm{OO}}\right\rangle\right)$ calculated for some selected internuclear distances for the $\mathrm{H}_{2}$ and $\mathrm{LiH}$ molecules. The present values obtained for $\mathrm{H}_{2}$ are compared with the results of Wolniewicz. ${ }^{12}$ All results are in hartrees.

\begin{tabular}{|c|c|c|c|c|c|c|c|}
\hline \multicolumn{5}{|c|}{$\mathrm{H}_{2}$} & \multicolumn{3}{|c|}{$\mathrm{LiH}$} \\
\hline \multirow{2}{*}{$\frac{\mathrm{R}}{0.4}$} & \multicolumn{2}{|c|}{$\mathrm{E}_{\text {nrel }}{ }^{12}$} & \multicolumn{2}{|c|}{$\left\langle\hat{H}_{\mathrm{OO}}\right\rangle^{12}$} & \multirow{2}{*}{$\frac{R}{2}$} & \multirow{2}{*}{$\begin{array}{c}\mathrm{E}_{\text {nrel }} \\
-8.0007611686\end{array}$} & \multirow{2}{*}{$\frac{\left\langle\hat{H}_{\mathrm{OO}}\right\rangle}{-0.356460}$} \\
\hline & -0.120230238 & -0.120230242 & -0.109059 & -0.109180 & & & \\
\hline 0.6 & -0.769635427 & -0.769635353 & -0.091518 & -0.091868 & 2.5 & -8.0582624519 & -0.399448 \\
\hline 0.8 & -1.020056664 & -1.020056603 & -0.076933 & -0.077003 & 3 & -8.0705406298 & -0.419094 \\
\hline 1 & -1.124539718 & -1.124539664 & -0.065105 & -0.065149 & 3.5 & -8.0647231889 & -0.428227 \\
\hline 1.4 & -1.174475714 & -1.174475671 & -0.047635 & -0.047666 & 4.1 & -8.0494548899 & -0.433039 \\
\hline 1.7 & -1.162458725 & -1.162458688 & -0.038209 & -0.038240 & 5 & -8.0244067293 & -0.435307 \\
\hline 2 & -1.138132954 & -1.138132919 & -0.030797 & -0.030821 & 6 & -8.0019762699 & -0.435635 \\
\hline 2.6 & -1.085791234 & -1.085791190 & -0.019659 & -0.019684 & 10 & -7.9785051124 & -0.435475 \\
\hline 6 & -1.000835703 & -1.000835702 & 0.000073 & 0.000082 & & & \\
\hline 8 & -1.000055603 & -1.000055603 & 0.000115 & 0.000142 & & & \\
\hline 10 & -1.000008754 & -1.000008754 & 0.000050 & 0.000131 & & & \\
\hline 19.5 & -1.000000124 & & 0.000003 & & & & \\
\hline
\end{tabular}

extensively optimized using the gradient-based procedure and the standard variational method. The energies obtained in the calculations are compared with the results of Wolniewicz ${ }^{12}$ in Table I. In general, our energies are slightly lower than those of Wolniewicz indicating a good quality of our electronic wave functions. These wave functions are used in the next step to calculate the expectation value of the orbit-orbit interaction operator. The results are also shown in Table I along with a comparison with the results obtained by Wolniewicz ${ }^{12}$ where Slater-type explicitly correlated functions expressed in terms of elliptic coordinates were used in expanding the wave function. In general, our results agree well with the results of Wolniewicz with the difference being in the 4-5 digits after the decimal point. Such a difference can be expected considering the difference in the basis set type and length. Based on the comparison, we conclude that the algorithm for calculating the expectation value of the OO operator with the wave function expanded in terms of $n$-electron ECGs is likely correct.

In the next step, to illustrate the capabilities of the present approach, calculations of the $\mathrm{OO}$ correction are performed for the four-electron $\mathrm{LiH}$ molecule and the two-electron $\mathrm{H}_{3}^{+}$ion. The $\mathrm{LiH}$ results are shown in Table $\mathrm{I}$ and those for $\mathrm{H}_{3}^{+}$are shown in Table II. For LiH, the OO correction is calculated at eight internuclear distances ranging from 2 to 10 a.u. and in the table its values are shown along with the total nonrelativistic energies. The calculations are performed employing the basis sets containing 2400 ECGs generated in Ref. 9 As there have not been any previous high-accuracy calculations of the OO correction for a four-electron molecular system before, no comparison can be made in this case. However, as the value of the correction changes by about $20 \%$ when

TABLE II. Non-relativistic ground-state energy and orbit-orbit relativistic correction calculated for the equilibrium geometry of $\mathrm{H}_{3}^{+}$(an equilateral triangle with each side equal to 1.65 a.u.). The present values are compared with the results of Cencek et al. ${ }^{7}$ All results are in hartrees.

\begin{tabular}{lllll}
\hline \hline $\mathrm{R}$ & \multicolumn{2}{c}{$\mathrm{E}_{\text {nrel }}{ }^{7}$} & $\left\langle\hat{H}_{\mathrm{OO}}\right\rangle^{7}$ \\
\hline 1.65 & -1.343835608 & -1.343835518 & -0.05721834 & -0.05721778 \\
\hline \hline
\end{tabular}

the internuclear distance increases from 2 to 10 a.u., it can be expected to have a non-negligible effect on the rovibrational transition energies when the correction is included in the LiH PES.

The illustration calculation for $\mathrm{H}_{3}^{+}$is done for the equilibrium geometry being an equilateral triangle with each side equal to 1.65 a.u. 900 fully optimized ECGs are used in the wave-function expansion. As one can see in Table II, the total nonrelativistic energy obtained in the calculation is lower than the energy obtained by Cencek et $a l .{ }^{7}$ The orbit-orbit correction of Cencek et al. ${ }^{7}$ agrees with our result within five significant figures. This provides additional validation of the algorithm implemented in this work.

Information of how the calculation of the OO matrix elements scales with the number of electrons and how it depends on the number of nuclei in the system may be interesting to the reader. The calculation of an OO matrix element involves multiplication of $n \times n$ matrices. This is an $n^{3}$ operation. In addition each matrix element includes a sum over the permutation-symmetry operators. There are $n$ ! of them. Thus the computation time of each matrix element is proportional to $n ! n^{3}$. The calculation of the whole OO matrix is quadratically dependent on the basis set size. Usually this size for a triatomic molecule needs to be somewhat larger than for a diatomic molecule with the same number of electrons in order to achieve similar accuracy in the calculations.

\section{CONCLUSION}

The development and computational implementation of the algorithm for calculating the orbit-orbit energy correction with all-electron ECGs pave the way for significantly improving the accuracy of the calculations of PESs for small molecules with two and more nuclei and two and more electrons. The calculations of rovibrational transition energies with such PESs can also be made significantly more accurate. With the nonrelativistic wave functions expanded in terms of a large number of all-electron 
ECGs whose non-linear parameters are extensively optimized using the gradient-based variational procedure, we expect to be now able to, for example, study upper regions of the rovibrational spectra of hydrogen-containing triatomics such as $\mathrm{H}_{3}^{+}, \mathrm{H}_{3}, \mathrm{HeH}_{2}^{+}$, and $\mathrm{LiH}_{2}^{+}$which so far eluded theoretical studies due to inadequate accuracy of the PES calculations.

\section{ACKNOWLEDGMENTS}

This work has been supported by the Polish National Science Centre, Grant No. DEC-2013/10/E/ST4/00033. Support by the National Science Foundation through Grant No. IIA-1444127 is also acknowledged.

${ }^{1}$ J. Tennyson, Astronomical Spectroscopy (Imperial College Press, 2005).

${ }^{2}$ N. R. Newbury and W. C. Swann, J. Opt. Soc. Am. B 24, 1756 (2007).
${ }^{3}$ M. Pavanello, L. Adamowicz, A. Alijah, N. F. Zobov, I. I. Mizus, O. L. Polyansky, J. Tennyson, T. Szidarovszky, and A. G. Csaszar, J. Chem. Phys. 136, 184303 (2012).

${ }^{4}$ M. Pavanello, L. Adamowicz, A. Alijah, N. F. Zobov, I. I. Mizus, O. L. Polyansky, J. Tennyson, T. Szidarovszky, A. G. Császár, M. Berg, A. Petrignani, and A. Wolf, Phys. Rev. Lett. 108, 023002 (2012).

${ }^{5}$ L. G. Diniz, J. R. Mohallem, A. Alijah, M. Pavanello, L. Adamowicz, O. L. Polyansky, and J. Tennyson, Phys. Rev. A 88, 032506 (2013).

${ }^{6}$ A. Petrignani, M. H. Berg, F. Grussie, A. Wolf, I. I. Mizus, O. L. Polyansky, J. Tennyson, N. F. Zobov, M. Pavanello, and L. Adamowicz, J. Chem. Phys. 141, 241104 (2014).

${ }^{7}$ W. Cencek, J. Rychlewski, R. Jaquet, and W. Kutzelnigg, J. Chem. Phys. 108, 2831 (1998).

${ }^{8}$ M. Stanke, E. Palikot, and L. Adamowicz, J. Chem. Phys. 144, 174101 (2016).

${ }^{9}$ W.-C. Tung, M. Pavanello, and L. Adamowicz, J. Chem. Phys. 134, 064117 (2011).

${ }^{10}$ S. Bubin, M. Cafiero, and L. Adamowicz, Adv. Chem. Phys. 131, 377 (2005).

${ }^{11}$ K. S. Miller, Math. Mag. 54, 67 (1981).

${ }^{12}$ L. Wolniewicz, J. Chem. Phys. 99, 1851 (1993). 